\title{
A new equation of state for core-collapse supernovae based on realistic nuclear forces and including a full nuclear ensemble
}

\author{
S. Furusawa ${ }^{1,2}$, H. Togashi ${ }^{3,4}$, H. Nagakura ${ }^{5}$, K. Sumiyoshi ${ }^{6}$, \\ S. Yamada ${ }^{7}$, H. Suzuki ${ }^{8}$ and M. Takano ${ }^{4,7}$ \\ ${ }^{1}$ Interdisciplinary Theoretical Science (iTHES) Research Group, RIKEN, Wako, \\ Saitama 351-0198, Japan \\ ${ }^{2}$ Frankfurt Institute for Advanced Studies, J.W. Goethe University, 60438 Frankfurt \\ am Main, Germany \\ ${ }^{3}$ RIKEN Nishina Center, RIKEN, Saitama 351-0198, Japan \\ ${ }^{4}$ Research Institute for Science and Engineering, Waseda University, Tokyo \\ 169-8555, Japan \\ ${ }^{5}$ TAPIR, Walter Burke Institute for Theoretical Physics, Mailcode 350-17, California \\ Institute of Technology, Pasadena, CA 91125, USA \\ ${ }^{6}$ Numazu College of Technology, Ooka 3600, Numazu, Shizuoka 410-8501, Japan \\ 7 Department of Science and Engineering, Waseda University, 3-4-1 Okubo, \\ Shinjuku, Tokyo 169-8555, Japan \\ ${ }^{8}$ Faculty of Science and Technology, Tokyo University of Science, Yamazaki 2641, \\ Noda, Chiba 278-8510, Japan
}

July 21,2017

\begin{abstract}
We have constructed a nuclear equation of state (EOS) that includes a full nuclear ensemble for use in core-collapse supernova simulations. It is based on the EOS for uniform nuclear matter that two of the authors derived recently, applying a variational method to realistic two- and there-body nuclear forces. We have extended the liquid drop model of heavy nuclei, utilizing the mass formula that accounts for the dependences of bulk, surface, Coulomb and shell energies on density and/or temperature. As for light nuclei, we employ a quantum-theoretical mass evaluation, which incorporates the Pauli- and self-energy shifts. In addition to realistic nuclear forces, the inclusion of in-medium effects on the full ensemble of nuclei makes the new EOS one of the most realistic EOS's, which covers a wide range of density, temperature and proton fraction that supernova simulations normally encounter. We make comparisons with the FYSS EOS, which is based on the same formulation for the nuclear ensemble but adopts the relativistic mean field (RMF) theory with the TM1 parameter set for uniform nuclear matter. The new EOS is softer than the FYSS EOS around and above nuclear saturation densities. We find that neutron-rich nuclei with small mass numbers are more abundant in the new EOS than in the FYSS EOS because of the larger saturation densities and smaller symmetry energy of nuclei in the former. We apply the two EOS's to 1D supernova simulations and find that the new EOS gives lower electron fractions and higher temperatures in the collapse phase owing to the smaller symmetry energy. As a result, the inner core has smaller masses for the new EOS. It is more compact, on the other hand, due to the softness of the new EOS and bounces at higher densities. It turns out that the shock wave generated
\end{abstract}


by core bounce is a bit stronger initially in the simulation with the new EOS. The ensuing outward propagations of the shock wave in the outer core are very similar in the two simulations, which may be an artifact, though, caused by the use of the same tabulated electron capture rates for heavy nuclei ignoring differences in the nuclear composition between the two EOS's in these computations.

\section{Introduction}

The nuclear equation of state (EOS) is an important ingredient to determine the dynamics of core collapse supernovae [1, 2, 3, 4]. It provides information on thermodynamical quantities such as pressure and composition of high-density matter realized in the supernova core, which is either a mixture of nuclei or uniformly distributed nucleons, for given baryon number density $n_{B}$, temperature $T$ and proton fraction

$Y_{p}$. The pressure is obviously a quantity that has a direct influence on the dynamics of supernova, determining, e.g., the strength of the shock wave at core bounce and the evolution of proto-neutron stars (PNS) in the post-bounce phase [5, 6, 7, 8, 9]. The abundance of various nuclei is important, affecting the neutronization of matter through electron captures on nuclei and the opacity for neutrino via the coherent scattering on heavy nuclei $[10,11,12]$. Note that the population of light nuclei such as deuteron, triton, helion and alpha particles may have some implications for shock revival $[13,14,15,16]$.

One of the critical elements in the construction of the nuclear EOS is the theoretical approach to nuclear interactions, on which the bulk properties of individual nuclei as well as the free energy of homogeneous nuclear matter depend strongly. In the previous EOS's used in supernova simulations, Skyrme type interactions [17] or meson-exchange models were adopted [18, 19, 20, 21, 22, 23, 24, 25, 26]. Both of them are based not on realistic interactions in vacuum that are known experimentally but on phenomenological medium-dependent interactions, whose model parameters are determined so that some nuclear properties such as the saturation density are reproduced. Recently, Togashi et al. [27] have constructed an EOS based on a realistic two-body nuclear potential, Argonne v18 [28], employing the variational method [29]. This is the first EOS of such kind, meant for supernova simulations.

The calculation of the EOS for homogeneous nuclear matter is not sufficient if the EOS were to be used in supernova simulations. We need in addition to treat inhomogeneous nuclear matter composed of various nuclei and dripped nucleons, which exists generically at sub-nuclear densities. The common choice is the so-called single nucleus approximation (SNA), in which the ensemble of heavy nuclei is represented just by a single, supposedly the most abundant nucleus. It was adopted in the two standard EOSs, widely employed in the simulations of core-collapse supernovae [17, 18, 19, 20] and also in the latest EOS [27]. Either the compressible liquid drop model (LDM) or the Thomas-Fermi approximation was employed in those EOS's. In such approximations, in-medium effects on the representative nucleus, such as compression, can be taken 
into account easily. Note that the abundance of various nuclei is important for the estimation of neutralization rates [30] and that it should be determined consistently with the modifications of nuclear properties in medium, since nuclear masses depends on the population of other nuclei whereas the nuclear abundance is dictated by the nuclear masses. It was demonstrated that the average mass number and the total mass fraction of heavy nuclei do not coincide with those of the representative nucleus in general $[31,32]$.

In the last few years, some research groups have constructed the multi-nuclear species EOS's [33, 34, 21, 35], in which the full ensemble of nuclei are taken into account with various finite-density and -temperature effects, such as the formation of nuclear pastas [36, 37, 38, 39, 40], smearing of shell effects in heavy nuclei [41, 42, 43, 44] and self- and Pauli-energy shifts in light nuclei [45, 46], being only partially considered. We have also constructed such an EOS (FYSS EOS) [24, 25, 26], in which the mass formula was extended for heavy nuclei and took a quantum approach for light clusters to accommodate all the in-medium effects mentioned above. Although the FYSS EOS may be better in the treatment of in-medium effects, it is sometimes pointed out that the relativistic mean field (RMF) with the TM1 parameter set [47], on which FYSS EOS is based, gives too high a symmetry energy $36.9 \mathrm{MeV}$ compared with the conventional values of $29.0-33.7 \mathrm{MeV}[48,49,50]$.

The purpose of the present study is to construct a new, multi-nuclear species EOS in the framework of the FYSS EOS but with a different EOS for uniform nuclear matter as a base. We use the EOS by Togashi et al. [29], who applied the variational method, one of the most popular techniques for the construction of the EOS of cold neutron star matter, using realistic nuclear forces expressed with the Argonne v18 potential [28]. Note that in their EOS the symmetry energy is $30.0 \mathrm{MeV}$, and with the inclusion of three-body forces, the saturation properties of uniform nuclear matter are satisfactorily reproduced. This is the first multi-nucleus EOS based on realistic nuclear forces. This article is organized as follows. In section 2, we describe the formulation adopted in this paper to construct the EOS. The results are shown in section 3 in comparison with the FYSS EOS derived from the RMF with the TM1 parameter set. We also compare the applications of these two EOS's to the one-dimensional supernova simulation in section 4 . The paper is wrapped up with a summary and some discussion in section 5 .

\section{Formulation}

The calculation below is based on the formulation of the FYSS EOS to describe the nuclear ensemble [24, 25, 26] and the variational principle method for uniform nuclear matter [29, 27]. For details, we refer the reader to Furusawa et al. [26] and Togashi et al. [29]. The free energy of the FYSS EOS is represented as

$$
f=f_{p, n}+\sum_{j} n_{j}\left(E_{j}^{t}+M_{j}\right)+\sum_{i} n_{i}\left(E_{i}^{t}+M_{i}\right),
$$


where $f_{p, n}$ is the free energy density of the free nucleons outside nuclei, $n_{j / i}$ are the number densities of individual light nuclei $j$ with the proton number $Z_{j} \leq 5$ and heavy nuclei $i$ with the proton number $6 \leq Z_{i} \leq 1000, E_{i / j}^{t}$ are the translational energies of heavy and light nuclei and $M_{i / j}$ are masses of heavy and light nuclei. In this work, the variational calculation takes the place of the RMF calculation in the previous model of the FYSS EOS, which provides the free energy density of nucleons $f_{p, n}$, the bulk energies of heavy nuclei in $M_{i}$ and the self-energy shifts of light nuclei in $M_{j}$.

\subsection{Free energy of free nucleons}

The variational calculations for the free energy of free nucleons are based on Refs. $[29,51,52]$. We start from the nuclear Hamiltonian composed of a two-body potential $V_{i j}$ and three-body potentials $V_{i j k}$, as in the Fermi Hypernetted Chain variational calculations [53]:

$$
H=-\sum_{i=1}^{N} \frac{\hbar^{2}}{2 m} \nabla^{2}+\sum_{i<j}^{N} V_{i j}+\sum_{i<j<k}^{N} V_{i j k},
$$

where $m$ is set to be the mass of neutron.

The free energy derived from two body interactions is obtained by using an extension of the variational method by Schmidt and Pandharipande $[54,55]$ with AV18 two-body potential [28] and the healing distance condition, which reproduces the internal energy per baryon of symmetric nuclear matter and neutron matter at zero temperature obtained by Akmal et al. [53]. The internal energy for three-body interactions is based on the UIX three-body potential $[56,57]$ with a phenomenological correction that reproduces empirical saturation properties. The entropy is expressed with average occupation probabilities of single quasi-nucleon states as in the case of a non-interacting Fermi gas [54]. The total free energy per baryon is minimized with respect to effective mases for nucleons. The optimized free energies agree reasonably with those of Akmal et al. [53] at zero temperature and those of Mukherjee [58] at finite temperature.

We account for the excluded-volume effect. The local number densities of free protons and neutrons are defined as $n_{p / n}^{\prime}=\left(N_{p / n}\right) /\left(V-V_{N}\right)$ where $V$ is the total volume, $V_{N}$ is the volume of all nuclei, and $N_{p / n}$ are the numbers of free protons and neutrons. The free energy density of free nucleons is given by

$$
f_{p, n}=\frac{\left(V-V_{N}\right)}{V}\left(n_{p}^{\prime}+n_{n}^{\prime}\right) \omega\left(T, n_{p}^{\prime}+n_{n}^{\prime}, n_{p}^{\prime} /\left(n_{p}^{\prime}+n_{n}^{\prime}\right)\right),
$$

where $\omega\left(T, n_{B}, x\right)$ is the free energy density per baryon in the unoccupied volume, $V-V_{N}$, for nucleons obtained from the variational calculation at temperature $T$, the local number density of baryon $n_{B}=n_{p}^{\prime}+n_{n}^{\prime}$ and the charge fraction $x=n_{p}^{\prime} /\left(n_{p}^{\prime}+n_{n}^{\prime}\right)$.

\subsection{Masses of heavy nuclei}

The masses of heavy nuclei are assumed to be the sum of the bulk, Coulomb, surface and shell energies: $M_{i}=E_{i}^{B}+E_{i}^{C}+E_{i}^{S f}+E_{i}^{S h}$. We define the saturation density of nuclei 
$n_{s i}(T)$ as the baryon number density, at which the free energy per baryon $\omega\left(T, n_{B}, Z_{i} / A_{i}\right)$ given by the variational method becomes a minimum, which is the same as that for the free energy density of free nucleons. Thus $n_{s i}(T)$ depends on the temperature $T$ and the charge fraction in each nucleus $Z_{i} / A_{i}$. At high temperatures $T \geq T_{c i}$, the free energy, $\omega\left(T, n_{B}, Z_{i} / A_{i}\right)$, has no minimum because of finite entropy. Then the saturation density $n_{s i}(T)$ above $T_{c i}$ is assumed to be equal to $n_{s i}\left(T_{c i}\right)$. When the saturation density $n_{s i}$ so obtained is lower than the baryon number density of the whole system $n_{B}$, we reset the saturation density as the baryon number density $n_{s i}=n_{B}$. This treatment of the saturation density is needed to obtain reasonable bulk energies at high temperatures and densities [25].

The bulk, surface, Coulomb and shell terms in $M_{i}$ are expressed as

$$
\begin{aligned}
& E_{i}^{B}=A_{i}\left\{\omega\left(T, n_{s i}, Z_{i} / A_{i}\right)\right\}, \\
& E_{i}^{C}= \begin{cases}\frac{3}{5}\left(\frac{3}{4 \pi}\right)^{-1 / 3} e^{2} n_{s i}^{2}\left(\frac{Z_{i}-n_{p}^{\prime} V_{i}^{N}}{A_{i}}\right)^{2} V_{i}^{N^{5 / 3}} D\left(u_{i}\right) & \left(u_{i} \leq 0.3\right), \\
\frac{3}{5}\left(\frac{3}{4 \pi}\right)^{-1 / 3} e^{2} n_{s i}^{2}\left(\frac{Z_{i}-n_{p}^{\prime} V_{i}^{N}}{A_{i}}\right)^{2} V_{i}^{B^{5 / 3}} D\left(1-u_{i}\right) & \left(u_{i} \geq 0.7\right),\end{cases} \\
& E_{i}^{S f}= \begin{cases}4 \pi r_{N i}^{2} \sigma_{i}\left(1-\frac{n_{p}^{\prime}+n_{n}^{\prime}}{n_{s i}}\right)^{2}\left(\frac{T_{c s}^{2}-T^{2}}{T_{c s}^{2}+T^{2}}\right)^{5 / 4} \quad\left(u_{i} \leq 0.3\right), \\
4 \pi r_{B i}^{2} \sigma_{i}\left(1-\frac{n_{p}^{\prime}+n_{n}^{\prime}}{n_{s i}}\right)^{2}\left(\frac{T_{c s}^{2}-T^{2}}{T_{c s}^{2}+T^{2}}\right)^{5 / 4} \quad\left(u_{i} \geq 0.7\right),\end{cases} \\
& E_{i}^{S h}= \begin{cases}E_{i 0}^{S h} \frac{\tau_{i}}{\sinh \tau_{i}} & \left(\rho \leq 10^{12} \mathrm{~g} / \mathrm{cm}^{3}\right), \\
E_{i 0}^{S h}\left(\frac{\tau_{i}}{\sinh \tau_{i}}\right)\left(\frac{\rho_{0}-\rho}{\rho_{0}-10^{12} \mathrm{~g} / \mathrm{cm}^{3}}\right) & \left(\rho>10^{12} \mathrm{~g} / \mathrm{cm}^{3}\right) .\end{cases}
\end{aligned}
$$

The bulk energy $E_{i}^{B}$ is evaluated from the free energy per baryon of uniform nuclear matter $\omega\left(T, n_{B}, x\right)$ given by the variational method at $n_{B}=n_{s i}$ and $x=Z_{i} / A_{i}$ for a given temperature $T$.

The Coulomb energy $E_{i}^{C}$ is obtained by integrating the Coulomb potential over the Wigner-Seitz cell containing nucleus $i$, dripped free protons and uniformly distributed electrons [24]. The cell is set to satisfy charge neutrality within the volume as $V_{i}=\left(Z_{i}-n_{p}^{\prime} V_{N i}\right) /\left(n_{e}-n_{p}^{\prime}\right)$, where $V_{N i}$ is the volume of the nucleus in the cell and can be calculated as $V_{N i}=A_{i} / n_{s i}$ and $n_{e}=Y_{p} n_{B}$ is the number density of electrons. The vapor volume and nucleus volume fraction in the cell are given by $V_{i}^{B}=V_{i}-V_{i}^{N}$ and $u_{i}=V_{i}^{N} / V_{i}$, respectively. Then $E_{i}^{C}$ is expressed as Eq. (5) where $D\left(u_{i}\right)=1-\frac{3}{2} u_{i}^{1 / 3}+\frac{1}{2} u_{i}$ and $e$ is the elementary charge. Each nucleus is assumed to enter the nuclear pasta phase individually when the volume fraction reaches $u_{i}=0.3$ and that the bubble shape is realized when it exceeds 0.7 .

The surface energy $E_{i}^{S f}$ is obtained from surface area times surface tension $\sigma_{i}=$ $\sigma_{0}-A_{i}^{2 / 3} /\left(4 \pi r_{N i}^{2}\right)\left[S_{s}\left(1-2\left(Z_{i} / A_{i}\right)^{2}\right)\right]$, where the values of the constants, $\sigma_{0}=1.15$ $\mathrm{MeV} / \mathrm{fm}^{3}$ and $S_{s}=45.8 \mathrm{MeV}$, are adopted from Lattimer and Swesty [17]. We may 
have to choose the values that are consistent with the EOSs for uniform nuclear matter. The simpler estimate, however, is adopted in this work, since there is no concrete way to derive the surface coefficients only from the EOS for uniform nuclear matter. The last two factors in Eq. (6) describe the reduction of the surface energy when the density contrast between the nucleus and the nucleon vapor decreases and when the temperature grows. The critical temperature is set to be $T_{c s}=18 \mathrm{MeV}$ [59] and the radii of nuclei and bubbles are expressed as $r_{N i}=\left(3 / 4 \pi V_{i}^{N}\right)^{1 / 3}$ and $r_{B i}=\left(3 / 4 \pi V_{i}^{B}\right)^{1 / 3}$. We use cubic polynomials of $u_{i}$ for the interpolation of Coulomb and surface energies between the droplet and bubble phases to ensure continuous and smooth connections at $u_{i}=0.3$ and $u_{i}=0.7$. We referred Watanabe et al. [36] for the criterion of intermediate states, although the thermodynamic quantities are hardly affected by its choice. The interpolation is mainly required to ensure the smooth change in mass fractions of nuclei $[25]$.

The shell energy at zero density and temperature is assumed to be the difference between the experimental or theoretical mass data [60, 61] and our liquid drop mass formula in the vacuum limit without the shell term: $E_{i 0}^{S h}=M_{i}^{\text {data }}-\left[E_{i}^{B}+E_{i}^{C}+\right.$ $\left.E_{i}^{S f}\right]_{T, n_{p / n}^{\prime}, n_{e}=0}$. The factor $\tau_{i} / \sinh \tau_{i}$ in Eq. (7) expresses the washout of shell effects approximately with $\tau_{i}=2 \pi^{2} T /\left(41 A_{i}^{-1 / 3}\right)$, which reproduces qualitatively the feature that shell effects disappear around $T \sim 2.03 .0 \mathrm{MeV}[26]$. The linear interpolation, $\left(\rho_{0}-\rho\right) /\left(\rho_{0}-10^{12} \mathrm{~g} / \mathrm{cm}^{3}\right)$, accounts for the disappearance of shell effects at high densities where $\rho$ and $\rho_{0}$ are the density and the saturation density, respectively [24].

\subsection{Masses of light nuclei}

We describe light nuclei as quasi-particles outside heavy nuclei, whose masses are assumed to be given by $M_{j}=M_{j}^{\text {data }}+\Delta E_{j}^{P a}+\Delta E_{j}^{S E}+\Delta E_{j}^{C}$. The Pauli-energy shift $\Delta E_{j}^{P a}$ for deuteron $(d)$, triton $(t)$, helion $(h)$ and alpha particle $(\alpha)$, which is fitted to the result of quantum statistical calculations [46], is given by [45]

$$
\begin{aligned}
& \Delta E_{j}^{P a}\left(n_{p l}, n_{n l}, T\right)=-\tilde{n}_{j}\left[1+\frac{\tilde{n}_{j}}{2 \tilde{n}_{j}^{0}(T)}\right] \delta B_{j}(T), \\
& \delta B_{j}(T)= \begin{cases}a_{j, 1} / T^{3 / 2}\left[1 / \sqrt{y_{j}}-\sqrt{\pi} a_{j, 3} \exp \left(a_{j, 3}^{2} y_{j}\right) \operatorname{erfc}\left(a_{j, 3} \sqrt{y_{j}}\right)\right] & \text { for } j=d, \\
a_{j, 1} /\left(T y_{j}\right)^{3 / 2} & \text { for } j=t, h, \alpha,\end{cases}
\end{aligned}
$$

where $\tilde{n}_{j}=2\left(Z_{j} n_{p l}+N_{j} n_{n l}\right) / A_{j}, y_{j}=1+a_{j, 2} / T, n_{p l / n l}$ are the local proton and neutron number densities that include light nuclei as well as free nucleons and $\tilde{n}_{j}^{0}(T)=B_{j}^{0} / \delta B_{j}(T)$ with the binding energy in vacuum, $B_{j}^{0}$. The Pauli-energy shifts for the light nuclei other than $d, t, h$ and $\alpha$ are calculated in the same way as that for $\alpha$.

The self-energy shift $\Delta E_{j}^{S E}$ is the sum of the self-energy shifts of individual nucleons in light nuclei $\Delta E_{n / p}^{S E}=\Sigma_{n / p}^{0}\left(T, n_{p}^{\prime}, n_{n}^{\prime}\right)-\Sigma_{n / p}\left(T, n_{p}^{\prime}, n_{n}^{\prime}\right)$ with $\Sigma^{0}$ and $\Sigma$ being the vector and scalar potentials of nucleon and the contribution from their effective masses $\Delta E_{j}^{\text {eff.mass }}=\left(1-m^{*} / m\right) s_{j}$ with $m^{*}=m_{B}-\Sigma_{n / p}\left(T, n_{p}^{\prime}, n_{n}^{\prime}\right)$ :

$$
\Delta E_{j}^{S E}\left(n_{p}^{\prime}, n_{n}^{\prime}, T\right)=\left(A_{j}-Z_{j}\right) \Delta E_{n}^{S E}+Z_{j} \Delta E_{p}^{S E}+\Delta E_{j}^{\text {eff.mass }} .
$$


For the potentials $\Sigma^{0}$ and $\Sigma$, we employ the parametric formula for RMF with the DD2 parameter set, Eqs. (A1) and (A2) in [45]. In the previous EOS [26], the potentials are evaluated consistently with the RMF with the TM1 parameter set employed for free nucleons. The self-energy shifts for the light nuclei other than $d, t, h$ and $\alpha$ are set to be zero. The parameters $a_{j / 1}, a_{j / 2}, a_{j / 3}$, and $s_{j}$ are given in Table I in [45]. The Coulomb energy shift is obtained from the same calculation for Coulomb energy of heavy nuclei, Eq. (5), by subtracting its vacuum limits although the nuclear pasta phases are not considered here. This mass evaluation for light clusters is stitched together by using a variety of different model assumptions, whereas mass fractions of light clusters in the FYSS EOS agree well with some experimental predictions $[62,50]$.

\subsection{Translational energies of nuclei and thermodynamical quantities}

The translational energy of nuclei is based on that for an ideal Boltzmann gas and is given by

$$
F_{i / j}^{t}=T\left\{\log \left(\frac{n_{i / j}}{g_{i / j}(T)\left(M_{i / j} T / 2 \pi \hbar^{2}\right)^{3 / 2}}\right)-1\right\}\left(1-\frac{n_{B}}{n_{s}}\right) .
$$

The last factor takes account of the excluded-volume effect in the same way as in Lattimer and Swesty [17]; each nucleus can move in the free space that is not occupied by baryons and its translational motion is suppressed at high densities. The internal degree of freedom $g_{i / j}(T)$ is defined as $g_{i}(T)=\left(g_{i}^{0}-1\right) \tau_{i} / \sinh \tau_{i}+1$ for heavy nuclei and $g_{j}(T)=g_{j}^{0}$ for light nuclei where the spin degree of freedom of the ground state is defined by $g_{i / j}^{0}$. The factor $\tau_{i} / \sinh \tau_{i}$ is incorporated washout of shell effects as in Eq. (7).

The abundances of nuclei as a function of $n_{B}, T$ and $Y_{p}$ are obtained by minimizing the model free energy in Eq. (1) with respect to the number densities of nuclei and nucleons under baryon and charge conservations. The nuclear abundance of each nucleus is determined by the nucleon chemical potentials $\mu_{p / n}$ and the nuclear mass $M_{i / j}$ under chemical equilibrium. Since both $\mu_{p / n}$ and $M_{i / j}$ depend on the local proton and neutron densities $n_{p / n}^{\prime}$ in our model, we solve the equations relating $\mu_{p / n}$ and $n_{p / n}^{\prime}$ as well as the two conservation equations to determine the four variables: $\mu_{p}, \mu_{n}, n_{p}^{\prime}$ and $n_{n}^{\prime}$.

Thermodynamical quantities other than the free energy are derived by partial differentiations of the optimized free energy density, in which all terms for in-medium effects are properly taken into account to ensure thermodynamical consistency. For example, the baryonic pressure and the entropy are obtained by the first derivative with respect to the baryonic density and the temperature, respectively.

\section{Results}

For comparison we present the results of the new EOS based on the variational method together with those of the previous FYSS EOS based on the RMF with the TM1 parameter set [26]. The free energy of free nucleons, bulk energy of heavy nuclei and selfenergy shifts of light nuclei are different between the two models. The shell, surface and 
Coulomb energies of heavy nuclei are also affected by the replacement of bulk properties such as the saturation densities of heavy nuclei. Table 1 summarizes the following bulk properties of nuclear matter at zero temperature: the nuclear saturation density of symmetric nuclear matter $n_{s 0}$, internal energy per baryon of symmetric matter at the saturation point $E_{0}$, compressibility $K$, symmetry energy $J$, and slope parameter in the density dependence of symmetry energy $L$. The variational EOS is softer with smaller values of $K, J$ and $L[27]$ although both EOS's can support the cold neutron stars of masses $\sim 2 M_{\odot}$ limit set by recent observations [63, 64]. On the other hand, radii of $1.4 M_{\odot}$ neutron stars are quite different and $\sim 14.2 \mathrm{~km}$ and $11.5 \mathrm{~km}$, for the RMF and variational EOS's, respectively [27].

Figure 1 compares the saturation densities $n_{s i}$ of heavy nuclei as a function of the charge fraction $Z_{i} / A_{i}$ at zero and critical temperatures, the latter of which correspond to the temperatures where the saturation disappears. The larger values of $J$ and $L$ in the FYSS EOS lead to much lower saturation densities for neutron-rich nuclei compared with the new EOS. Not only the bulk energy but also the Coulomb and surface energies depend on $n_{s i}$.

Figure 2 displays the individual values of the bulk, shell, Coulomb, surface and binding energies per baryon of nuclei with $A_{i}=100$ at two temperatures. Note that the sign is changed in the former two and the baryon rest mass is subtracted from the bulk energy, which is defined as $E_{i}^{\prime B}$, and the binding energy per baryon is expressed as $B_{i}=-\left(E_{i}^{\prime B}+E_{i}^{C}+E_{i}^{S f}+E_{i}^{S h}\right) / A_{i}$. The larger $n_{s i}$ in the new EOS results in smaller surface energies and larger Coulomb energies of heavy nuclei. Neutron-rich nuclei in the FYSS EOS have smaller absolute values of the bulk energy due to the larger values of $J$ and $L$. At the higher temperature $(T \gtrsim 3 \mathrm{MeV})$, absolute values of the bulk energy become larger due to the entropy contribution from nucleons in the nucleus, which increases as the saturation density is reduced, especially for the FYSS EOS.

Below we present the results for $T=1,3$ and $5 \mathrm{MeV}$ and $Y_{p}=0.2$ and 0.4, typical values in the supernova core. Figure 3 shows the mass fractions of free protons and neutrons, and of light and heavy nuclei. For the neutron-rich conditions of $Y_{p}=0.2$, the mass fraction of heavy nuclei in the new EOS is larger than that in the FYSS EOS. This is because neutron-rich nuclei have larger binding energies or smaller masses. As for $Y_{p}=0.4$, the difference between the two EOS's is small compared with the neuron-rich conditions, since the bulk properties in the asymmetric nuclear matter are not much different between them. We find that the mass fraction of light nuclei decreases at some density and the heavy nuclei take their place at higher densities in both EOS's. The mass fraction of free neutrons in the new EOS tends to be smaller than in the FYSS EOS due to the smaller value of $J$ in the former, which leads to large populations of neutron-rich nuclei. The mass fractions of free protons, on the other hand, are almost the same between the two EOS's.

Figure 4 displays the critical lines, where the mass fractions of heavy nuclei $\left(Z_{i} \geq 6\right)$, $X_{A}=\sum_{i} A_{i} n_{i} / n_{B}$ and of light nuclei $\left(Z_{j}<6\right), X_{a}=\sum_{j} A_{j} n_{j}$, become $10^{-4}$. At low temperatures $(T \lesssim 3 \mathrm{MeV})$, heavy nuclei appear at lower densities in the new EOS 
than in the FYSS EOS whereas, at high temperatures, the order of the critical densities becomes opposite, since the RMF we use yields larger absolute values of the bulk energy, which originate from the entropy contributions of nucleons in nuclei. On the other hand, both EOS's give similar critical densities in Fig. 4, at which light nuclei show up. Note also the densities, at which nuclear pastas melt into uniform nuclear matter depend on the saturation densities and those in the FYSS EOS are smaller.

Figure 5 shows the mass fractions of the isotopes of $\mathrm{Ni}\left(Z_{i}=28\right)$ and $\mathrm{Sn}\left(Z_{i}=50\right)$. In all cases considered there, larger amounts of neutron-rich isotopes appear in the new EOS than in the FYSS EOS due to the smaller symmetry energy. Figure 6, on the other hand, displays the mass number distributions of heavy nuclei for four different conditions. At $n_{B}=10^{-3} \mathrm{fm}^{-3}, T=1 \mathrm{MeV}$ and $Y_{p}=0.2$, nuclei with large mass numbers are more abundant in the new EOS, since the smaller symmetry energy allows the nuclei in the vicinity of the neutron magic number $N=126$ to be more populated, which can also be confirmed in Fig. 5. For the other three conditions, the mass distribution in the new EOS shifts slightly leftward compared with the FYSS EOS owing to the smaller surface and larger Coulomb energies that in turn result from the larger saturation densities in the former EOS. The difference between the two EOS's is particularly small at $T=1 \mathrm{MeV}$ and $Y_{p}=0.4$, which can be confirmed both in Figs. 5 and 6 . Note that zig-zag features remarkable at $T=1 \mathrm{MeV}$, for which the shell effects are responsible, are almost washed out at $T=3 \mathrm{MeV}$.

Figure 7 exhibits the average mass numbers of heavy nuclei as a function of density and indicates that they are smaller in the new EOS than in the FYSS EOS in most cases due to the larger saturation densities in the former. At $n_{B} \lesssim 10^{-2} \mathrm{fm}^{-3}, T=1$ $\mathrm{MeV}$ and $Y_{p}=0.2$, the result is the other way round because of the larger abundance of neutron-rich magic nuclei for the new EOS.

Finally we compare thermodynamical quantities of relevance. Figures 8-10 present the free energy per baryon, baryonic pressure and entropy per baryon, respectively. Around the saturation density, the FYSS EOS based on the RMF gives much larger free energies and baryon pressures for all combinations of density, temperature and proton fractions considered there due to its stiffer bulk property. On the other hand, the difference of the free energies is not significant at low densities. At low temperatures and high densities, the pressures become negative, since the Coulomb energies of heavy nuclei is decreased by the rise of electron density [24]. The densities, at which the pressure becomes negative, are influenced by the Coulomb energy and the nuclear composition and are hence a bit different between the two EOS's. Note that the total pressure of supernova matter is always positive owing to electron's contribution [21]. The entropy per baryon is slightly affected by the difference in the mass fractions of nucleons and nuclei as displayed in Fig. 3. 


\section{Applications to 1D simulations of core-collapse supernovae}

Implementing the new EOS in our neutrino radiation-hydrodynamics code, we have conducted spherically symmetric simulations of core collapse for a $11.2 M_{\odot}$ supernova progenitor [65]. We have paid particular attention to the difference from the FYSS EOS [26], with which we perform a comparable simulation with the same setting. We designate the simulation with the new EOS as the VM model and the one with the FYSS EOS as the RMF model.

Our code solves simultaneously the Boltzmann equations for neutrino transport, hydrodynamic equations for matter motions and Poisson equations for Newtonian gravity, since they are all coupled with one another. We follow the dynamics up to $150 \mathrm{~ms}$ after core bounce. Neutrino-matter interactions are treated in the same way as in Nagakura et al. [66, 67], which include updated sets of weak interactions and of electron capture (EC) rates for heavy nuclei among other things [68, 69, 70]. They are calculated based on the nuclear populations obtained from a nuclear statistical equilibrium EOS and with the previous version of the FYSS EOS [25] and tabulated as functions of $\rho, T$ and $Y_{p}$. Note that the same table is used for the simulations with the new and FYSS EOS's. It is true that the use of the EC rates based on the nuclear abundances derived with each EOS's is more consistent in these simulations, but we are concerned with the difference not in the EC rates but in the EOS's themselves in this paper. We therefore decided to use the same EC table in this study. The results of fully consistent core collapse simulations will be reported elsewhere soon. For more technical details of our code, we refer readers to Nagakura et al. [66].

It turns out that the overall dynamics are not much different between the two simulations. In particular, the shock radius is surprisingly similar to each other as a function of time even though the distributions of $\rho, T$ and $Y_{p}$ are not so identical as described in detail below. This seems to be an accident, with different effects canceling each other. The use of the same EC rates may also be contributing to this similarity. In neither case, we obtain an explosion as expected for spherical models. If one looks into details, however, some differences become apparent, to which we shift our attention hereafter.

In Fig. 11, we display radial profiles of several important quantities as a function of mass coordinate at three different times in the collapse phase, i.e., when the central density $\left(\rho_{c}\right)$ reaches $10^{11}, 10^{12}$ and $10^{13} \mathrm{~g} / \mathrm{cm}^{3}$. In these figures, we can immediately recognize the differences in the temperature and entropy profiles in the central region between the two models, which are particularly significant after the central density reaches $10^{12} \mathrm{~g} / \mathrm{cm}^{3}$. This higher entropy for the new EOS originates from smaller mass fractions of heavy nuclei in the core matter with $Y_{p} \sim 0.46$ at the beginning of the collapse. The reason is as follows: in the matter with heavy nuclei and $\alpha$ particles dominating over free nucleons, the mass fraction of heavy nuclei is approximately given as $X_{A} \sim\left(0.5-Y_{p}\right) /(0.5-\bar{Z} / \bar{A})$, which is in turn obtained from the baryon number conservation $X_{A}+X_{\alpha} \sim 1$ and the charge neutrality $(\bar{Z} / \bar{A}) X_{A}+0.5 X_{\alpha} \sim Y_{e}=Y_{p}$, 
where $\bar{Z}$ and $\bar{A}$ are the average atomic and mass numbers of heavy nuclei. For a given $Y_{p}$, neutron-rich nuclei are more abundant, implying a smaller $\bar{Z} / \bar{A}$, in the new EOS, because of the smaller symmetry energy as discussed in the previous section. This is borne out in the bottom left panel of Fig. 11. The matter around the mass coordinate of $1.0 M_{\odot}$ is similar to the matter at the center at the initial time. It is evident that the VM model gives a smaller $X_{A}$ and a larger $X_{a} \sim X_{\alpha}$, and hence a higher entropy per baryon although $\rho, T$ and $Y_{p}$ are almost identical between the two models. In the early phase of core collapse $\left(\rho_{c} \sim 10^{10} \mathrm{~g} / \mathrm{cm}^{3}\right)$, the temperature of the central region increases more rapidly by adiabatic compression in the VM model with the higher entropy than in the RMF model. The higher temperature accelerates electron captures and deleptonizes the core more quickly (see the radial profile of $Y_{e}$ in Fig. 11). The smaller electron fraction decreases the degeneracy pressure of electrons, accelerating the collapse, raising the temperature further, and resulting in rather large deviations in temperature and entropy (see the corresponding panels). Note that the entropy is changed by electron captures and accompanied emissions of neutrinos.

It is also important to note that such high temperatures and small electron fractions reduce the mass fraction of heavy nuclei further, enhancing the mass fractions of nucleons and light nuclei instead. The matter in the central region $\left(\lesssim 0.6 M_{\odot}\right)$ consists of heavy nuclei with $\bar{A} \gtrsim 60$, light nuclei and dripped neutrons when $\rho_{c}$ exceeds $\sim 10^{12} \mathrm{~g} / \mathrm{cm}^{3}$. For such matter with $T \lesssim 3 \mathrm{MeV}$ and $Y_{p}\left(=Y_{e}\right) \lesssim 0.4$, the new EOS, in contrast to the previous case, tends to give greater values to $X_{A}$ than the FYSS EOS, as shown in the previous section. Owing to the higher $T$ and lower $Y_{p}\left(=Y_{e}\right)$ in the VM model than in the RMF model, however, the order is reserved, keeping $X_{A}$ lower in the dynamical simulations of the VM model. The smaller mass fractions of heavy nuclei imply lower opacities from neutrino-nucleus coherent scatterings, the dominant source of opacity during collapse, meaning that the deleptonization is further accelerated in the VM model. This in turn makes the mass of the inner core smaller. As is well known, the small mass of the inner core is disadvantageous for the outward propagation of the shock wave generated by core bounce, since the gravitational energy liberated and hence the initial shock energy tend to be smaller, and the outer core, the obstacle for shock propagation, becomes larger [71, 72].

Figure 12 shows the radial profiles of the same quantities as in Fig. 11 but at the time of core bounce, which we define in this study as the time when the shock wave reaches the mass coordinate of $0.6 M_{\odot}$. As discussed in the previous paragraph, the stronger deleptonization for the VM model gives the central electron fraction $\sim 0.25$ at bounce, which is $\sim 10 \%$ smaller than that for the RMF model. We find, however, that the shock wave is stronger in the VM model than in the RMF, which can be understood from the greater jump of entropy at the shock wave for the VM model. This result may seem at odds with our previous statement that the shock energy tends to be lower for the smaller inner core. This is true only when the EOS is identical, though. In fact, the new EOS is softer than the FYSS EOS as mentioned earlier. Then the inner core becomes not only less massive but also more compact, which is corroborated in the top 
left panel of the figure. The central density at core bounce is indeed higher in the VM model. The smaller radius of the inner core means a larger liberation of gravitational energy. Indeed, this is sufficient to compensate for the smaller mass of inner core and leads to the similar trajectory of shock wave at later times in the two models (see the right panel in Fig. 13).

The left panel in Fig. 13 compares the time evolutions of the central density in the post-bounce phase between the two models. The new EOS has consistently higher values than the RMF model, an indication that the former is softer and the inner core is more compact. In spite of the rather large difference in the central density between the two models, the time evolutions of the shock radii are quite similar to each other (see the right panel in the same figure). It should be noted, however, that this coincidence might be an artifact of our use of the same EC rates for heavy nuclei, which is certainly inconsistent in the VM and RMF models, not correctly reflecting the differences in the nuclear composition. In fact, the outer cores have very similar deleptonization histories during the collapsing phase between the two models (see the panel for $Y_{e}$ in Fig. 11), which means that the shock waves propagate through very similar outer cores. It is also important to note that the luminosity and mean energy of neutrinos are also almost identical between the two models (see Fig. 14), meaning that neutrino coolings are almost the same, which also contributes to the similar evolutions of shock radius.

It is interesting to note that substantial amounts of heavy nuclei exist around the PNS (see the left panel in Fig. 15) and they survive for more than $100 \mathrm{~ms}$ after bounce (see the upper left panel in Fig. 16). We expect that they might affect the diffusion of neutrinos, since the coherent scattering by heavy nuclei gives far greater opacities than scatterings on free nucleons or light nuclei. Incidentally, there is a non-negligible mass fraction of light nuclei just behind the shock wave (see the right panel of Fig. 15). This may indicate that weak interactions between neutrinos and these light nuclei should not be ignored in the post-shock region and may play an important role in the timing of the neutrino burst. We will address this issue by incorporating the relevant weak interactions with light nuclei in simulations $[14,15,16]$. It should be noted that nonnegligible mass fractions of light nuclei appear just outside the PNS. In fact, their mass fraction sometimes overwhelms that of nucleons. These light nuclei, both behind the shock wave and near the PNS, may hence have the potential to change the neutrino cooling/heating (see also [14]) and affect shock revival eventually. The detailed study on these effects will be presented in our forthcoming paper.

\section{Summary and discussion}

We have constructed a new equation of state for use in supernova simulations. It includes the full ensemble of nuclei and is derived from the EOS for uniform nuclear matter that employed realistic nuclear forces in the variational method. This should be contrasted with standard EOS's, which include a single representative nucleus and treat the nuclear interactions phenomenologically with either Skyrme type interactions 
or meson-exchange models. In the calculation of nuclear masses, we have taken into consideration various in-medium effects such as the smearing of shell effects in heavy nuclei and the self- and Pauli-energy shifts in light nuclei at high densities and/or temperatures.

For some representative conditions in supernovae, we have compared the new EOS and our previous one: the FYSS EOS, in which the RMF EOS with the TM1 parameter set is employed. At $T=1 \mathrm{MeV}$ and $Y_{p}=0.4$, typical values at the center in the initial phase of collapse, the difference between the nuclear compositions in the two EOS's is not so large, since normal nuclear statistical equilibrium is expected to prevail with negligible in-medium effects. For more neutron-rich conditions $\left(Y_{p} \sim 0.2\right)$, the smaller symmetry energy in the new EOS provides larger mass fractions of heavy nuclei than the FYSS EOS. At higher temperatures $(T \gtrsim 3 \mathrm{MeV})$, the larger entropy contribution leads to larger total mass fraction of heavy nuclei in the FYSS EOS than in the new EOS. It is also found that the new EOS tends to have larger mass fractions of nuclei with small mass numbers and charge fractions owing to the larger saturation densities and the smaller symmetry energy. The abundance of light nuclei, on the other hand, is not much changed by the replacement of the previous EOS with the new one although it is somewhat affected by the difference in the mass fractions of heavy nuclei. This is because in the new EOS the self- and Pauli-energy shifts are not evaluated consistently with the EOS for uniform nuclear matter.

Employing the two EOS's, we have performed dynamical simulations of corecollapse under the assumption of spherical symmetry. We have found that the new EOS gives smaller mass fractions of heavy nuclei and higher entropies for conditions typical in the early phase of core-collapse. This is due to the lower symmetry energy in the new EOS, which leads to slightly lower values of the charge fraction. Owing to higher entropies, the temperature rises more quickly in the simulation with the new EOS. The higher temperatures then enhance electron captures, resulting in lower electron fractions and even higher temperatures as the collapse proceeds. As a result, the mass fraction of heavy nuclei continues to be lower at later times in the collapsing phase, where $Y_{e}$ becomes much lower with the new EOS than with the FYSS EOS in spite of the fact that the new EOS tends to give larger mass fractions of heavy nuclei in neutron-rich matter. The reduction of electron fraction implies a smaller mass of the inner core.

The lower electron fractions obtained in the simulation with the new EOS imply that the mass of the inner core is smaller. On the other hand, the inner core is more compact for the new EOS, with the central density being higher. This is due to the fact that the new EOS is softer than the FYSS EOS around nuclear density. These two effects, i.e., the smaller mass and the higher density of the inner core tend to work oppositely for the initial strength of the shock wave generated by core bounce, with the former (latter) reducing (enhancing) the shock strength. As a consequence, the shock is a bit stronger initially for the new EOS than for the FYSS EOS. It turns out, however, that the ensuing outward propagations of the shock wave in the outer core are not much different between the two EOS's. This may be an artifact, though, as the 
same EC rates for heavy nuclei were used in spite of different compositions in the two simulations. Indeed the deleptonization may be more suppressed for the new EOS, since neutron-rich nuclei, which have smaller EC rates in general, are more abundant in the new EOS. The results of more consistent simulations will be reported in our forthcoming paper.

Very recently, three of the authors of this paper published a paper on another EOS for supernova simulations, which we refer to as the Togashi EOS [27]. It is derived from the same EOS obtained with the variational method for uniform nuclear matter by Togashi et al. [29] and employs the SNA based on the Thomas-Fermi approximation. As expected, the differences between our new EOS and the Togashi EOS are very similar to those observed between the FYSS and STOS EOS's [18, 19, 20]. Details are discussed in Furusawa et al. [24]. As a matter of fact, the latter two EOS's employ the same RMF EOS for uniform nuclear matter but at sub-nuclear densities the former handles the ensemble of nuclei just as the new EOS does whereas the latter resorts to the SNA that the Togashi EOS adopts. The multi-nuclear species EOS's, i.e., the new EOS and the FYSS EOS, commonly predict smaller atomic and mass numbers than SNA EOS's, or the Togashi EOS and the STOS EOS [31, 24, 32]. In the new EOS and the FYSS EOS, shell effects are taken into account and, as a result, the atomic and mass numbers grow with density in a step-wise fashion while they increase smoothly in the STOS and Togashi EOS's, in which shell effects are neglected. Furthermore, the entropy per baryon is lower in the latter two EOS's because they ignore the translational motion of the representative nucleus [21, 24].

Although the major differences between the new EOS and the Togashi EOS are mostly ascribed to the SNA adopted in the latter, there are certainly other differences. In fact, the differences between the STOS and Togashi's EOS's are not always consistent with those between the FYSS EOS and the new EOS's. For instance, the Togashi EOS predicts larger mass numbers than the STOS EOS for neutron-rich conditions $\left(Y_{p} \sim 0.1\right)$ because of the larger saturation density in the former, which leads to greater surface energy described by gradient terms in the energy functional employed in the ThomasFermi calculation. On the other hand, the new EOS gives smaller mass numbers than the FYSS EOS more often than not although there is no general trend in part due to the shell effects. This happens because we use the same coefficient for surface tensions, $\sigma_{0}$, in the new EOS and the FYSS EOS, which was originally adopted in the LDM [17]. Then, the surface energy becomes smaller in the new EOS with the larger saturation density. It is true that the surface tension should be treated in a consistent manner with the EOS for uniform nuclear matter but the difference that the surface energy would make is far smaller than that stemming from the SNA approximation discussed noted in the previous paragraph.

Note that the mass models of heavy and light nuclei employed in the new EOS still have some room for improvement, since there are uncertainties in our phenomenological treatment of in-medium effects. In addition, in the new EOS we have adopted the same parameters in the formulae for the shell and surface energies of heavy nuclei as 
well as for the Pauli energies of light nuclei as those employed in the FYSS EOS. They should be changed according to the theory used to describe uniform nuclear matter. The light clusters, especially deuterons, in the new EOS may have to be described in the framework of the variational method by using realistic nuclear forces. Nevertheless, we believe that the new EOS is one of the most elaborate EOS's constructed so far for supernova simulations. The tabulated new EOS is available in the public domain $\dagger$.

\section{Acknowledgments}

S. F. and H. N. were supported by Japan Society for the Promotion of Science Postdoctoral Fellowships for Research Abroad. Some numerical calculations were carried out on the PC cluster at the Center for Computational Astrophysics, National Astronomical Observatory of Japan. This work was supported by the RIKEN iTHES Project and in part by the usage of supercomputer systems through the Large Scale Simulation Program (Nos. 15/16/-08,16/17-11) of High Energy Accelerator Research Organization (KEK) and Post-K Projects (hp 150225, hp160071, hp160211,hp170031,hp170230) at K-computer, RIKEN AICS as well as the computational resources provided by RCNP at Osaka University, YITP at Kyoto University, University of Tokyo and JLDG. This work was supported by a Grant-inAid for the Scientific Research from the Ministry of Education, Culture, Sports, Science and Technology (MEXT), Japan (24103006, 24244036, 16H03986, 15K05093, 24105008, $25400275,26104006)$. 
[1] Janka H T 2012 Annual Review of Nuclear and Particle Science 62 407-451

[2] Kotake K, Takiwaki T, Suwa Y, Iwakami Nakano W, Kawagoe S, Masada Y and Fujimoto S i 2012 Advances in Astronomy 2012428757

[3] Burrows A 2013 Reviews of Modern Physics 85 245-261

[4] Foglizzo T, Kazeroni R, Guilet J, Masset F, González M, Krueger B K, Novak J, Oertel M, Margueron J, Faure J, Martin N, Blottiau P, Peres B and Durand G 2015 Publications of the Astronomical Society of Australia 32 e009

[5] Sumiyoshi K, Yamada S, Suzuki H, Shen H, Chiba S and Toki H 2005 Astrophys. J. 629 922-932

[6] Marek A, Janka H T and Müller E 2009 Astronomy \& Astrophysics 496 475-494

[7] Suwa Y, Takiwaki T, Kotake K, Fischer T, Liebendörfer M and Sato K 2013 Astrophys. J. 764 99

[8] Fischer T, Hempel M, Sagert I, Suwa Y and Schaffner-Bielich J 2014 Eur. Phys. J. A50 46

[9] Togashi H, Takano M, Sumiyoshi K and Nakazato K 2014 Progress of Theoretical and Experimental Physics 2014 023D05

[10] Hix W R, Messer O E, Mezzacappa A, Liebendörfer M, Sampaio J, Langanke K, Dean D J and Martínez-Pinedo G 2003 Physical Review Letters 91201102

[11] Lentz E J, Mezzacappa A, Messer O E B, Hix W R and Bruenn S W 2012 Astrophys. J. 76094

[12] Sullivan C, O'Connor E, Zegers R G T, Grubb T and Austin S M 2016 Astrophys. J. 81644

[13] Sumiyoshi K and Röpke G 2008 Phys. Rev. C 77(5) 055804

[14] Furusawa S, Nagakura H, Sumiyoshi K and Yamada S 2013 Astrophys. J. 77478

[15] Nasu S, Nakamura S X, Sumiyoshi K, Sato T, Myhrer F and Kubodera K 2015 Astrophys. J. 80178

[16] Fischer T, Martínez-Pinedo G, Hempel M, Huther L, Röpke G, Typel S and Lohs A 2016 Expected impact from weak reactions with light nuclei in corecollapse supernova simulations European Physical Journal Web of Conferences (European Physical Journal Web of Conferences vol 109) p 06002

[17] Lattimer J M and Swesty F D 1991 Nuclear Physics A 535 331-376

[18] Shen H, Toki H, Oyamatsu K and Sumiyoshi K 1998 Nuclear Physics A 637 435-450

[19] Shen H, Toki H, Oyamatsu K and Sumiyoshi K 1998 Progress of Theoretical Physics 100 1013-1031

[20] Shen H, Toki H, Oyamatsu K and Sumiyoshi K 2011 Astrophys.J.Suppl. 19720

[21] Hempel M and Schaffner-Bielich J 2010 Nuclear Physics A 837 210-254

[22] Steiner A W, Hempel M and Fischer T 2013 Astrophys. J. 77417

[23] Shen G, Horowitz C J and Teige S 2011 Phys. Rev. C 83035802

[24] Furusawa S, Yamada S, Sumiyoshi K and Suzuki H 2011 Astrophys. J. 738178

[25] Furusawa S, Sumiyoshi K, Yamada S and Suzuki H 2013 Astrophys. J. 77295

[26] Furusawa S, Sumiyoshi K, Yamada S and Suzuki H 2017 Nuclear Physics A 957188 - 207 ISSN 0375-9474

[27] Togashi H, Nakazato K, Takehara Y, Yamamuro S, Suzuki H and Takano M 2017 Nuclear Physics A 96178 - 105 ISSN 0375-9474

[28] Wiringa R B, Stoks V G J and Schiavilla R 1995 Phys. Rev. C 51(1) 38-51

[29] Togashi H and Takano M 2013 Nuclear Physics A 902 53-73

[30] Furusawa S, Nagakura H, Sumiyoshi K, Kato C and Yamada S 2017 Phys. Rev. C 95(2) 025809

[31] Burrows A and Lattimer J M 1984 Astrophys. J. 285 294-303

[32] Furusawa S and Mishustin I 2017 Phys. Rev. C 95(3) 035802

[33] Botvina A S and Mishustin I N 2004 Physics Letters B 584 233-240

[34] Botvina A S and Mishustin I N 2010 Nuclear Physics A 843 98-132

[35] Buyukcizmeci N, Botvina A S and Mishustin I N 2014 Astrophys. J. 78933

[36] Watanabe G, Maruyama T, Sato K, Yasuoka K and Ebisuzaki T 2005 Physical Review Letters 94 031101

[37] Newton W G and Stone J R 2009 Phys. Rev. C 79055801

[38] Okamoto M, Maruyama T, Yabana K and Tatsumi T 2012 Physics Letters B 713 284-288 
[39] Schneider A S, Horowitz C J, Hughto J and Berry D K 2013 Phys. Rev. C 88(6) 065807

[40] Horowitz C J, Berry D K, Caplan M E, Fischer T, Lin Z, Newton W G, O'Connor E and Roberts L F 2016 ArXiv e-prints (Preprint 1611.10226)

[41] Brack M and Quentin P 1974 Physics Letters B 52 159-162

[42] Bohr A and Mottelson B 1998 Nuclear Structure (Nuclear Structure no v. 2) (World Scientific) ISBN 9789810239800

[43] Sandulescu N, Civitarese O, Liotta R J and Vertse T 1997 Phys. Rev. C 55 1250-1254

[44] Nishimura S and Takano M 2014 Shell effects in hot nuclei and their influence on nuclear composition in supernova matter American Institute of Physics Conference Series (American Institute of Physics Conference Series vol 1594) ed Jeong S, Imai N, Miyatake H and Kajino T pp 239-244

[45] Typel S, Röpke G, Klähn T, Blaschke D and Wolter H H 2010 Phys. Rev. C 81015803

[46] Röpke G 2009 Phys. Rev. C 79014002

[47] Sugahara Y and Toki H 1994 Nucl.Phys. A579 557-572

[48] Lattimer J M and Lim Y 2013 Astrophys. J. 77151

[49] Danielewicz P and Lee J 2014 Nuclear Physics A 9221 - 70 ISSN 0375-9474

[50] Oertel M, Hempel M, Klähn T and Typel S 2017 Reviews of Modern Physics 89015007

[51] Kanzawa H, Oyamatsu K, Sumiyoshi K and Takano M 2007 Nuclear Physics A 791 232-250

[52] Kanzawa H, Takano M, Oyamatsu K and Sumiyoshi K 2009 Prog. Theor. Phys. 122673

[53] Akmal A, Pandharipande V R and Ravenhall D G 1998 Phys. Rev. C 58 1804-1828

[54] Schmidt K E and Pandharipande V R 1979 Physics Letters B 87 11-14

[55] Mukherjee A and Pandharipande V R 2007 Phys. Rev. C 75035802

[56] Carlson J, Pandharipande V R and Wiringa R B 1983 Nuclear Physics A 401 59-85

[57] Pudliner B S, Pandharipande V R, Carlson J and Wiringa R B 1995 Phys. Rev. Lett. 74(22) 4396-4399

[58] Mukherjee A 2009 Phys. Rev. C 79(4) 045811

[59] Bondorf J P, Botvina A S, Iljinov A S, Mishustin I N and Sneppen K 1995 Physics Reports 257 $133-221$

[60] Audi G, Wang M, Wapstra A, Kondev F, MacCormick M, Xu X and Pfeiffer B 2012 Chinese Physics C 361287

[61] Koura H, Tachibana T, Uno M and Yamada M 2005 Progress of Theoretical Physics 113 305-325

[62] Hempel M, Hagel K, Natowitz J, Röpke G and Typel S 2015 Phys. Rev. C 91(4) 045805

[63] Demorest P B, Pennucci T, Ransom S M, Roberts M S E and Hessels J W T 2010 Nature 467 1081-1083

[64] Antoniadis J, Freire P C C, Wex N, Tauris T M, Lynch R S, van Kerkwijk M H, Kramer M, Bassa C, Dhillon V S, Driebe T, Hessels J W T, Kaspi V M, Kondratiev V I, Langer N, Marsh T R, McLaughlin M A, Pennucci T T, Ransom S M, Stairs I H, van Leeuwen J, Verbiest J P W and Whelan D G 2013 Science 340448

[65] Woosley S E, Heger A and Weaver T A 2002 Rev. Mod. Phys. 74(4) 1015-1071

[66] Nagakura H, Iwakami W, Furusawa S, Sumiyoshi K, Yamada S, Matsufuru H and Imakura A 2017 The Astrophysical Journal Supplement Series $\mathbf{2 2 9} 42$

[67] Nagakura H, Iwakami W, Furusawa S, Okawa H, Harada A, Sumiyoshi K, Yamada S, Matsufuru H and Imakura A 2017 ArXiv e-prints (Preprint 1702.01752)

[68] Juodagalvis A, Langanke K, Hix W R, Martínez-Pinedo G and Sampaio J M 2010 Nuclear Physics A $848454-478$

[69] Langanke K and Martínez-Pinedo G 2000 Nuclear Physics A 673 481-508

[70] Langanke K, Martínez-Pinedo G, Sampaio J M, Dean D J, Hix W R, Messer O E B, Mezzacappa A, Liebendörfer M, Janka H T and Rampp M 2003 Phys. Rev. Lett. 90(24) 241102

[71] Yahil A and Lattimer J M 1982 Supernovae for pedestrians NATO Advanced Science Institutes (ASI) Series C (NATO Advanced Science Institutes (ASI) Series C vol 90) ed Rees M J and Stoneham R J pp 53-70 
[72] Bruenn S W 1985 Astrophys.J.Suppl 58 771-841 


\begin{tabular}{|c||c|c|c|c|c|}
\hline model & $n_{s 0}\left(\mathrm{fm}^{-3}\right)$ & $E_{0}(\mathrm{MeV})$ & $K(\mathrm{MeV})$ & $J(\mathrm{MeV})$ & $L(\mathrm{MeV})$ \\
\hline RMF TM1 & 0.145 & -16.3 & 281 & 36.9 & 110.8 \\
\hline Variational method & 0.160 & -16.0 & 245 & 30.0 & 35.0 \\
\hline
\end{tabular}

Table 1. Bulk properties of nuclear matter obtained in the two EOS's for uniform nuclear matter. See the beginning of Sec. 3 .

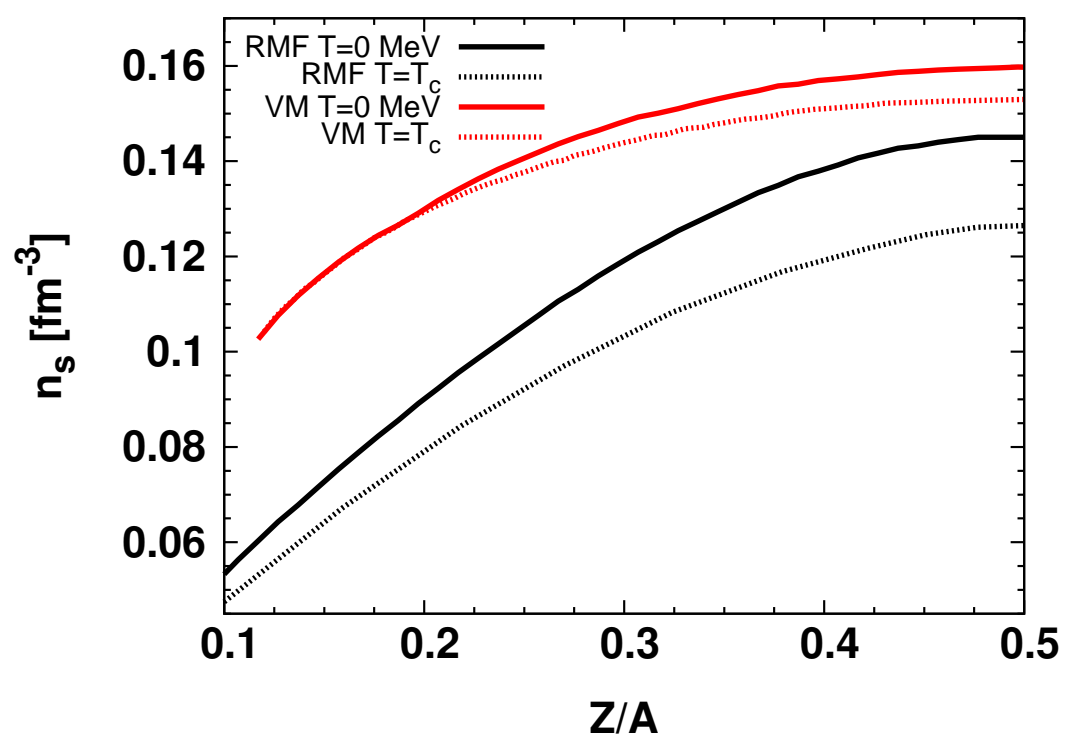

Figure 1. Saturation densities at zero temperature (solid lines) and those at critical temperatures (dashed lines) as a function of charge fraction for the models based on the variational method (red lines) and the RMF (black lines) 

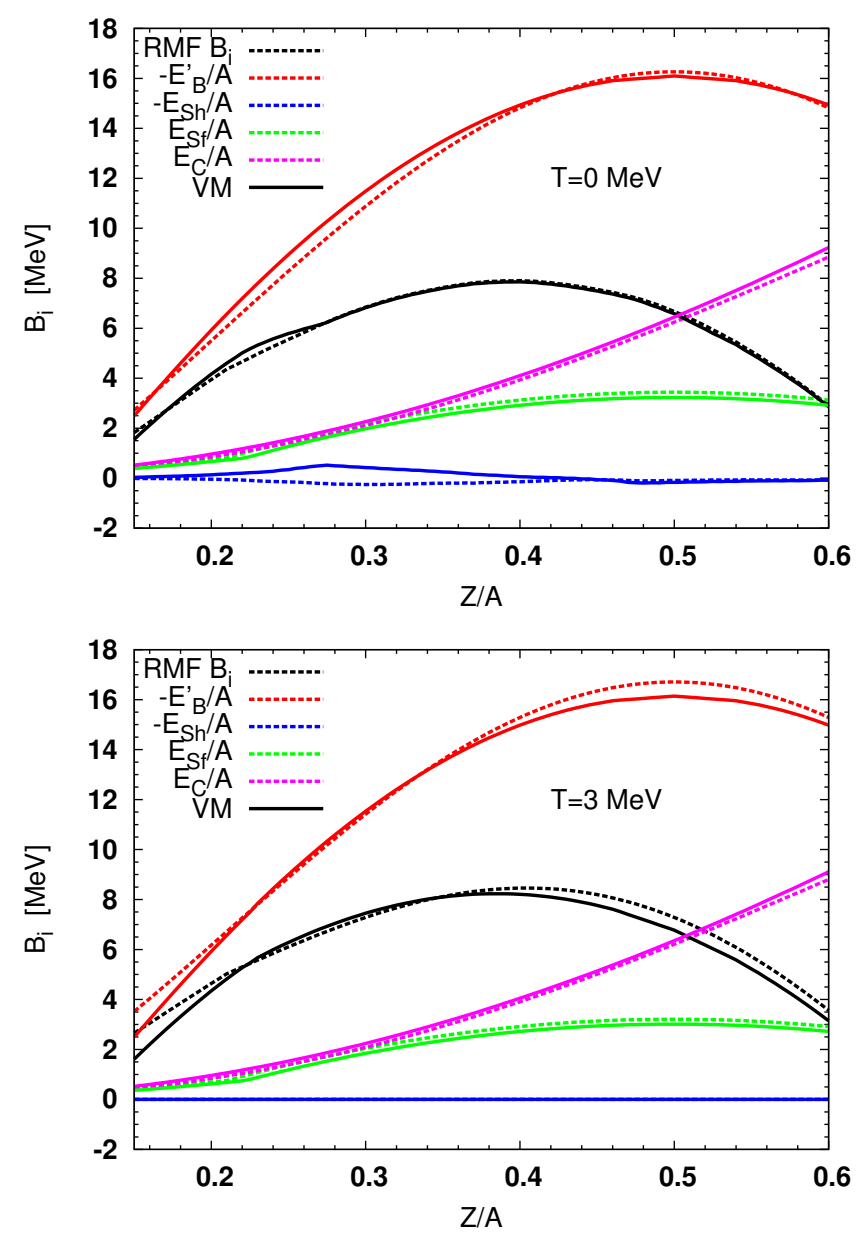

Figure 2. Binding (black lines), surface (green lines), Coulomb (magenta lines), bulk (red lines) and shell (blue lines) energies per baryon of the nuclei with $A_{i}=100$ at the vanishing density and $T=0 \mathrm{MeV}$ (left panel) and $3 \mathrm{MeV}$ (right panel) as a function of charge fraction for the new EOS (solid lines) and the FYSS EOS (dashed lines). Note that the sign of the bulk and shell energies is changed for convenience. 

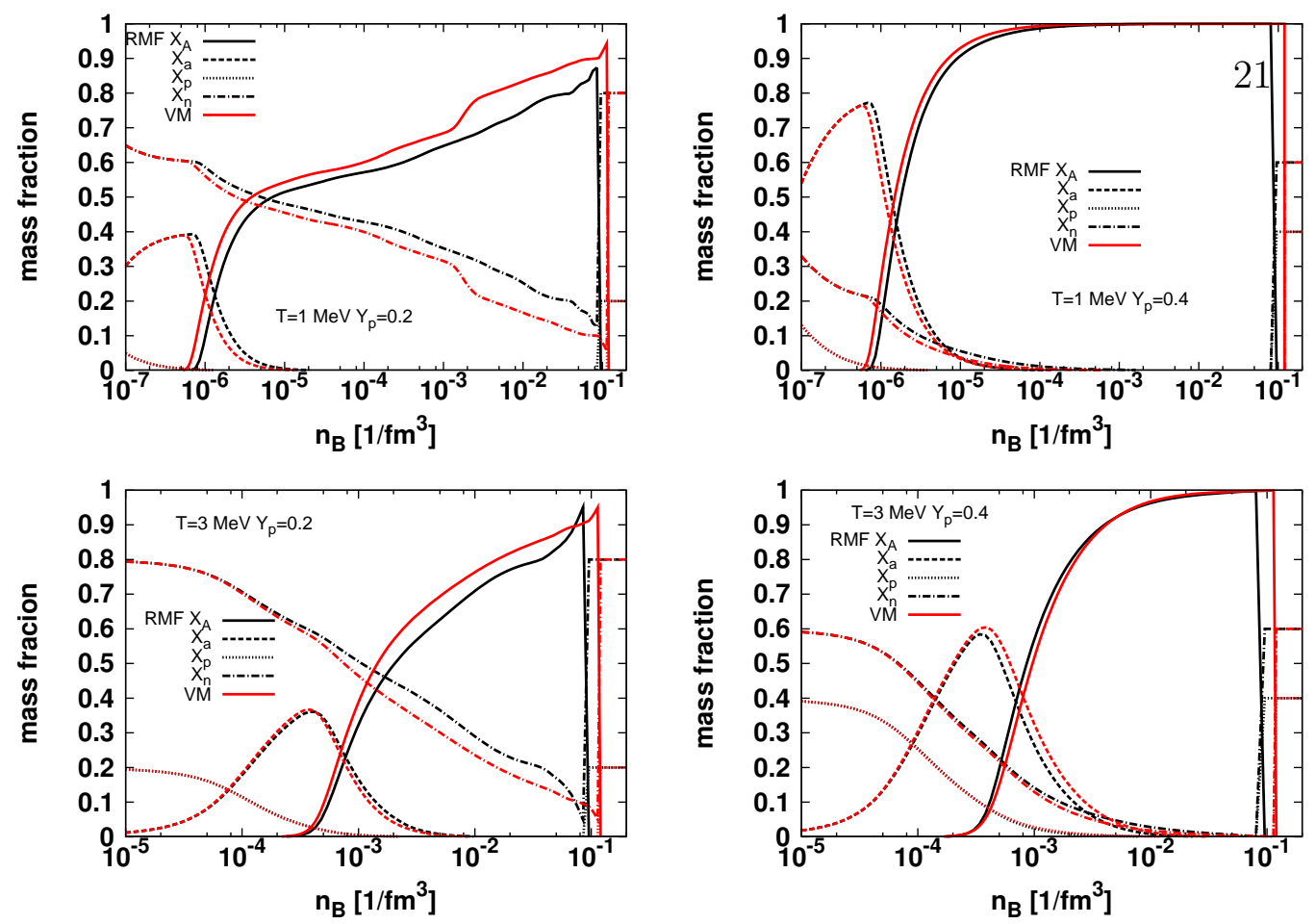

Figure 3. Mass fractions of heavy nuclei with $Z_{i} \geq 6$ (solid lines), $X_{A}$, of light nuclei with $Z_{i} \leq 5$ (dashed lines), $X_{a}$, of free neutrons (dashed dotted lines), $X_{n}$, and of free protons (dotted lines), $X_{p}$, for the new EOS (red lines) and FYSS EOS (black lines) as a function of baryon number density, $n_{B}$, at $T=1 \mathrm{MeV}$ (top row) and $3 \mathrm{MeV}$ (bottom row) and $Y_{p}=0.2$ (left column) and 0.4 (right column). 

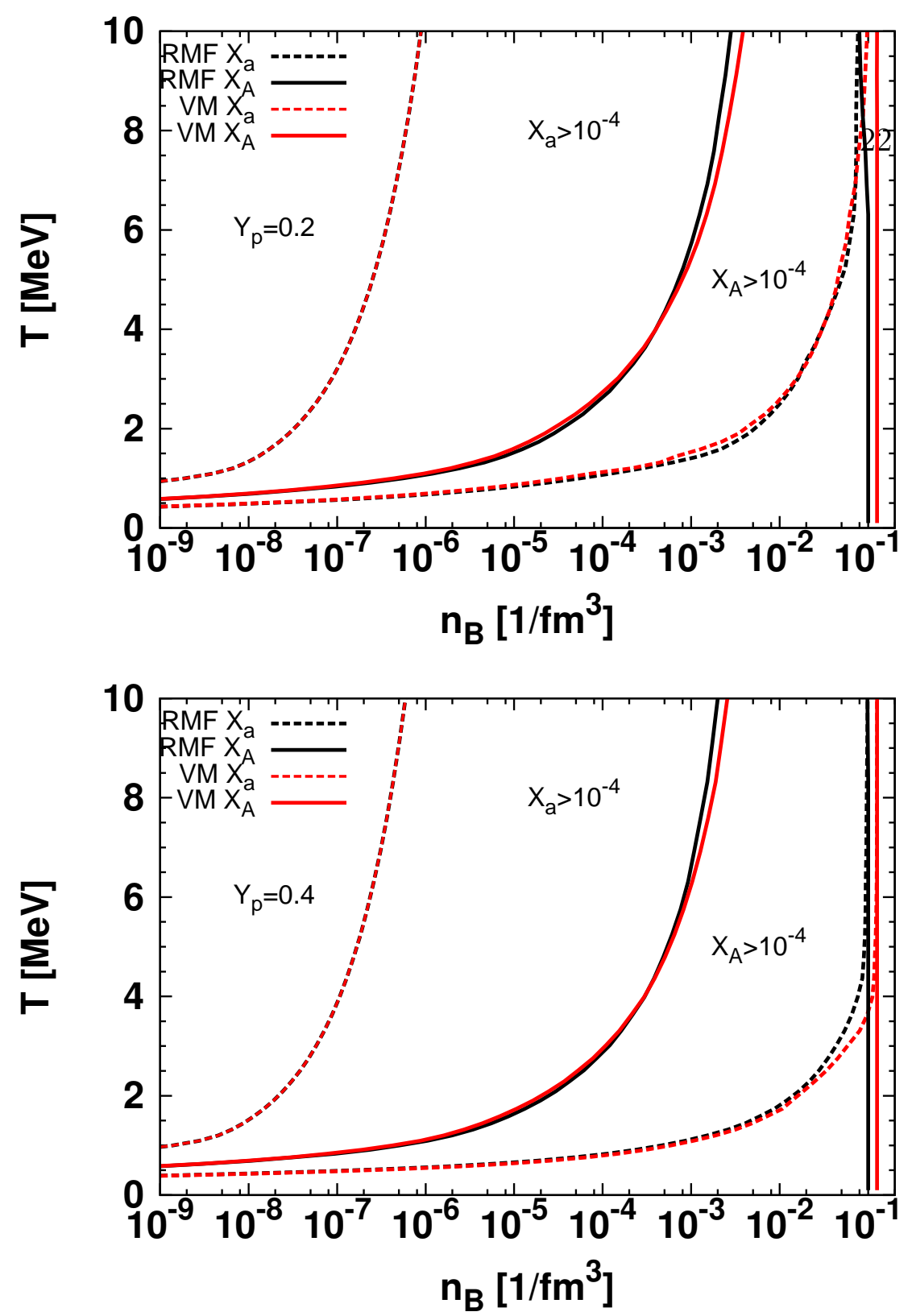

Figure 4. Critical lines, on which the mass fractions of light and heavy nuclei become $X_{a}=10^{-4}$ and $X_{A}=10^{-4}$, for the new EOS (red lines) and the FYSS EOS (black lines) at $Y_{p}=0.2$ (top panel) and 0.4 (bottom panel). 

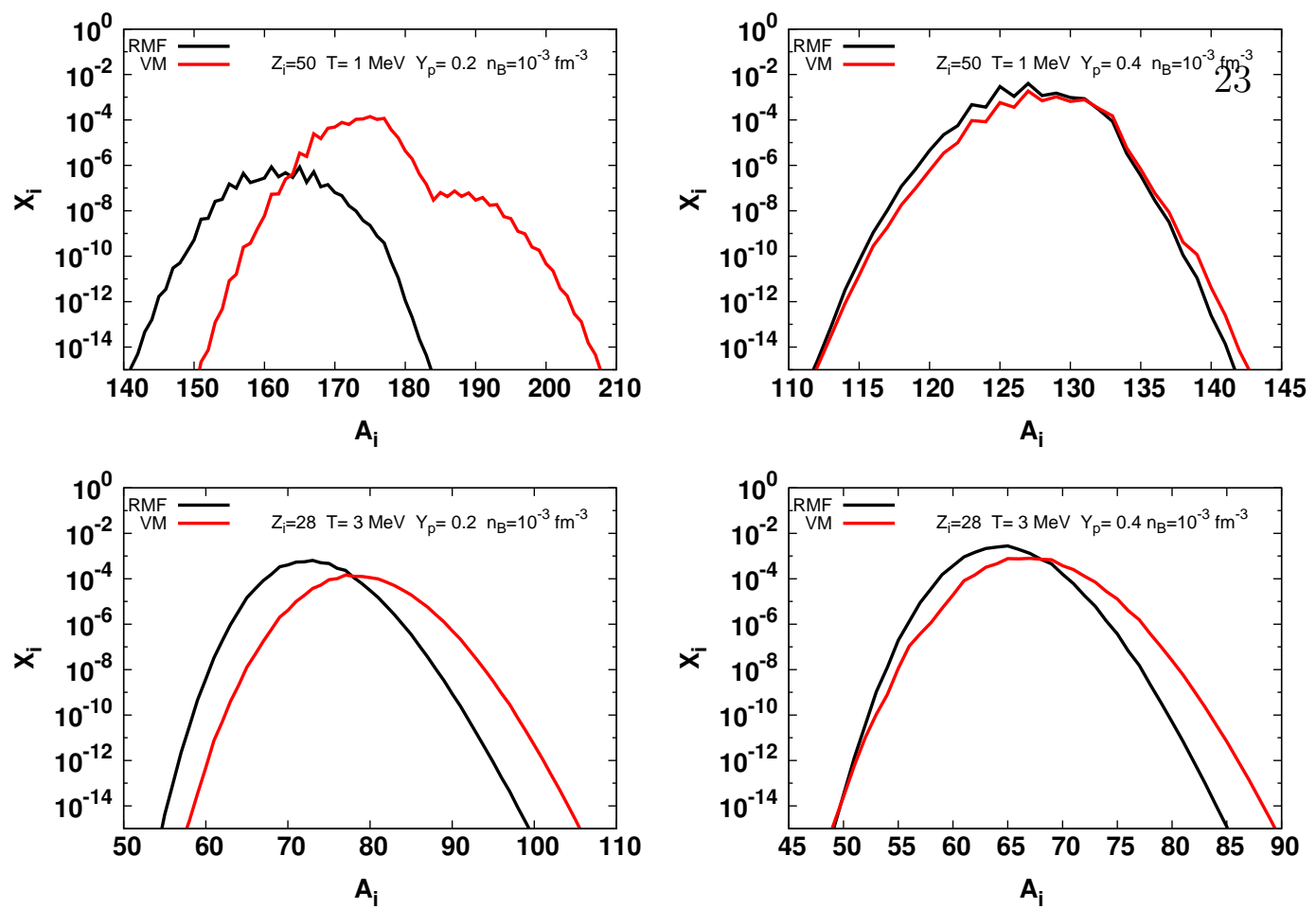

Figure 5. Mass fractions of the isotopes of $\mathrm{Sn}\left(Z_{i}=50\right)$ for $T=1 \mathrm{MeV}$ (top row) and of $\mathrm{Ni}\left(Z_{i}=28\right)$ for $T=3 \mathrm{MeV}$ (bottom row) as a function of mass number for the new EOS (red lines) and the FYSS EOS (black lines) at $Y_{p}=0.2$ (left column) and 0.4 (right column) and $n_{B}=10^{-3} \mathrm{fm}^{-3}$. 

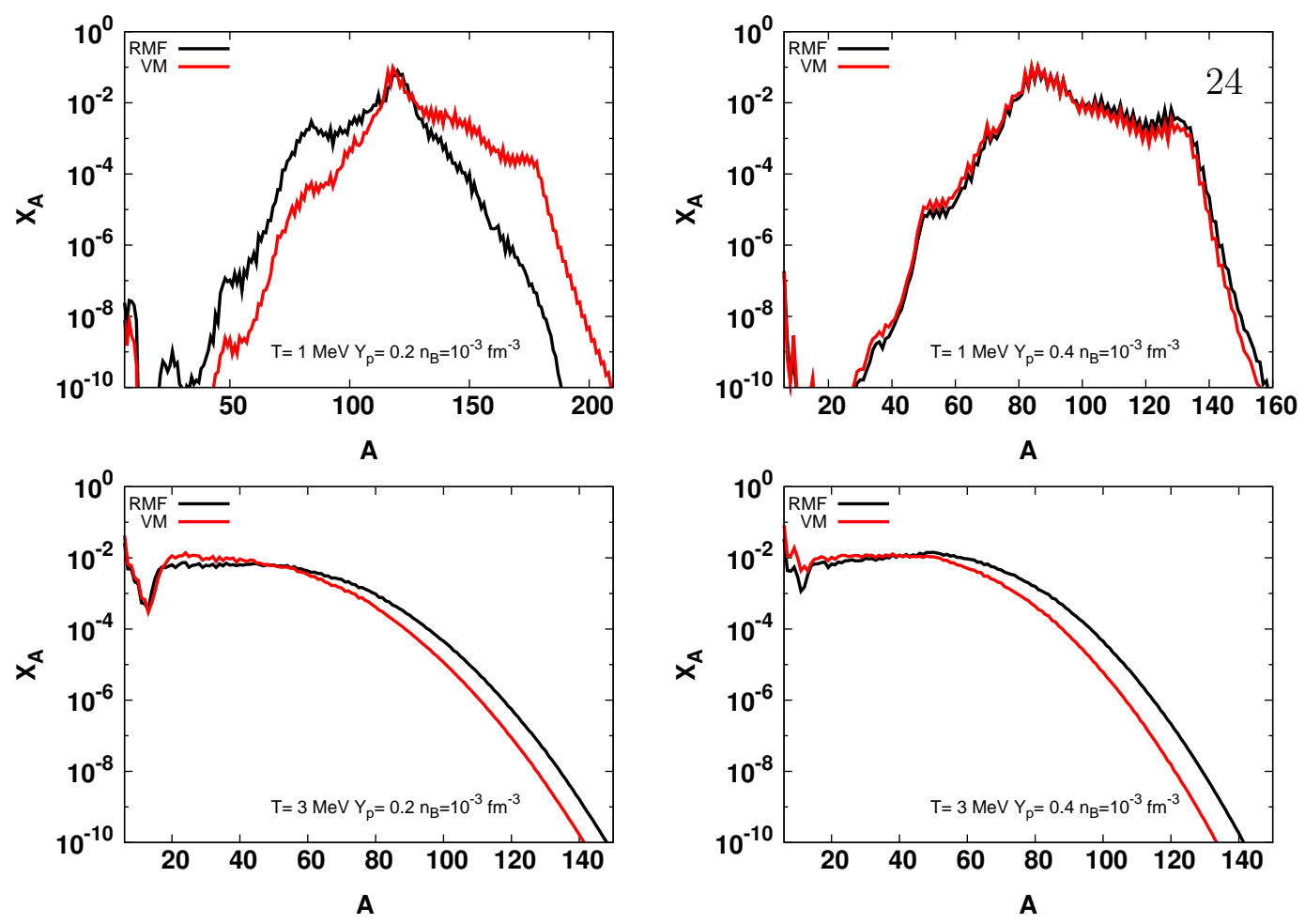

Figure 6. Size distributions of heavy nuclei as a function of mass number for the new EOS (red lines) and the FYSS EOS (black lines) at $T=1 \mathrm{MeV}$ (top row) and $3 \mathrm{MeV}$ (bottom row), $Y_{p}=0.2$ (left column) and 0.4 (right column) and $n_{B}=10^{-3} \mathrm{fm}^{-3}$.
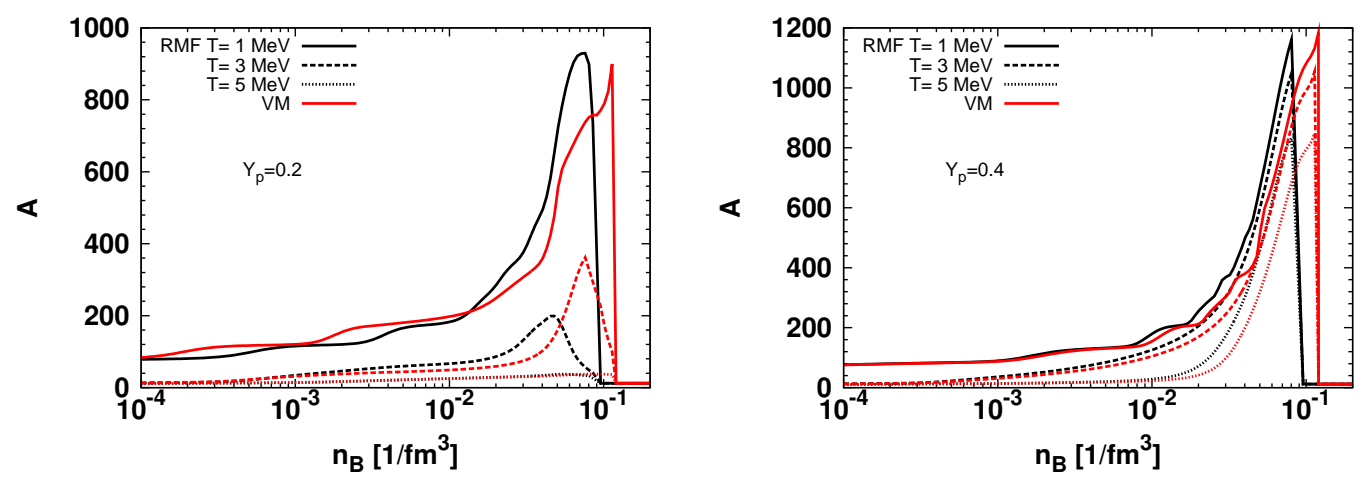

Figure 7. Average mass numbers of heavy nuclei with $Z \geq 6$ as a function of $n_{B}$ for the new EOS (red lines) and the FYSS EOS (black lines) at $T=1 \mathrm{MeV}$ (solid lines), $3 \mathrm{MeV}$ (dashed lines) and $5 \mathrm{MeV}$ (dotted lines) for $Y_{p}=0.2$ (left panel) and 0.4 (right panel). 

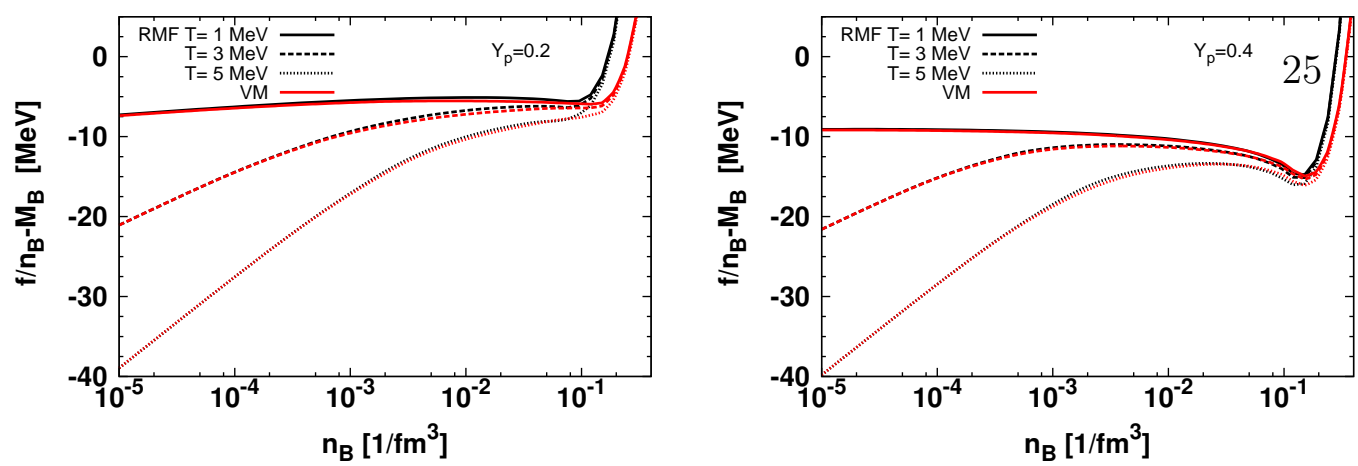

Figure 8. Free energies per baryon relative to the baryon rest mass as a function of $n_{B}$ for the new EOS (red lines) and the FYSS EOS (black lines) at $T=1 \mathrm{MeV}$ (solid lines), $3 \mathrm{MeV}$ (dashed lines) and $5 \mathrm{MeV}$ (dotted lines) for $Y_{p}=0.2$ (left panel) and 0.4 (right panel).
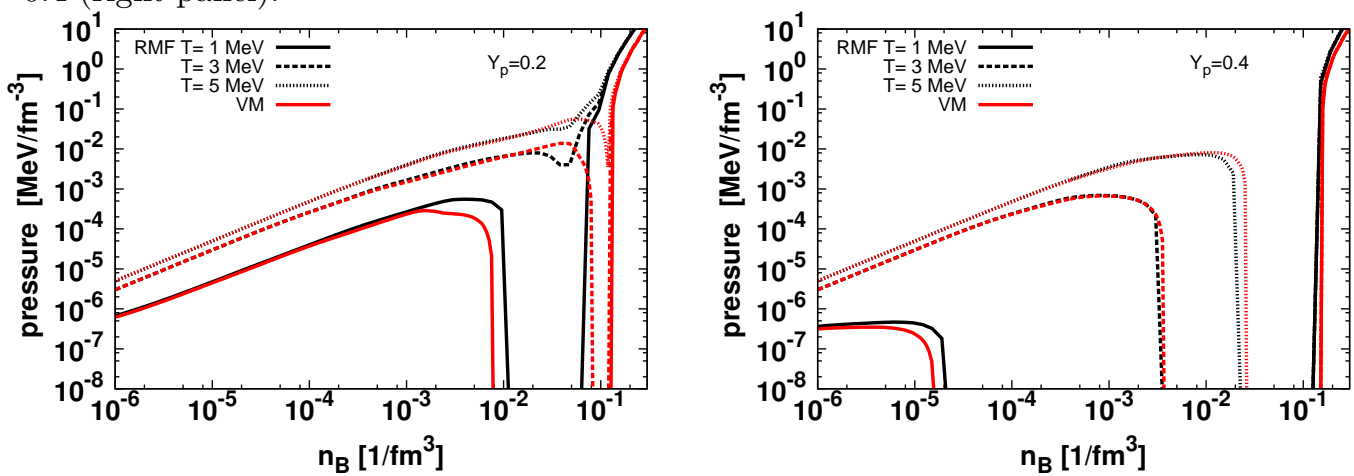

Figure 9. Baryonic pressures as a function of $n_{B}$ for the new EOS (red lines) and the FYSS EOS (black lines) at $T=1 \mathrm{MeV}$ (solid lines), $3 \mathrm{MeV}$ (dashed lines) and 5 $\mathrm{MeV}$ (dotted lines) for $Y_{p}=0.2$ (left panel) and 0.4 (right panel).
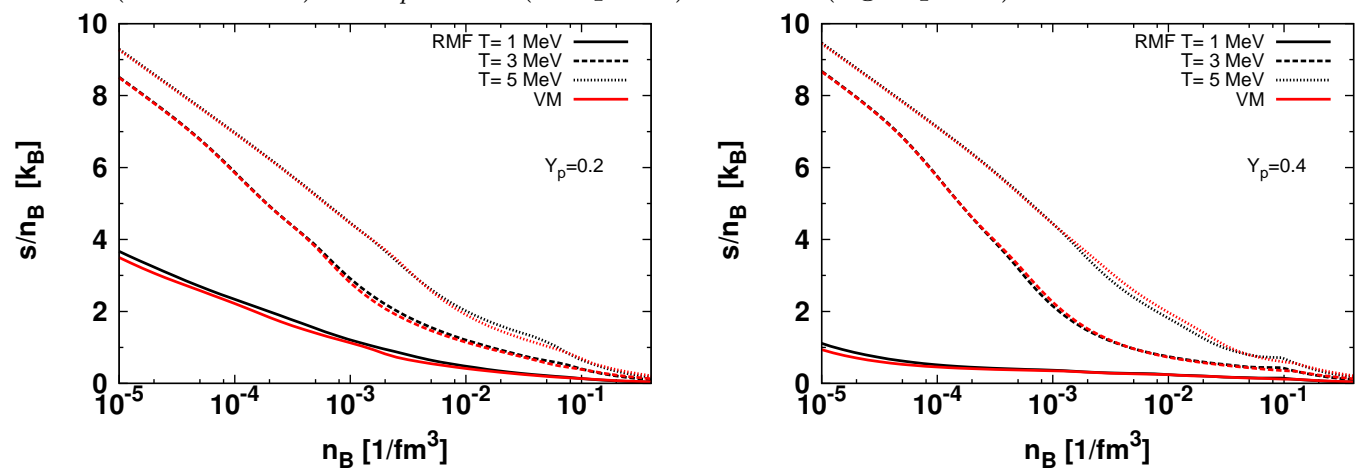

Figure 10. Entropies per baryon as a function of $n_{B}$ for the new EOS (red lines) and the FYSS EOS (black lines) at $T=1 \mathrm{MeV}$ (solid lines), $3 \mathrm{MeV}$ (dashed lines) and $5 \mathrm{MeV}$ (dotted lines) for $Y_{p}=0.2$ (left panel) and 0.4 (right panel). 

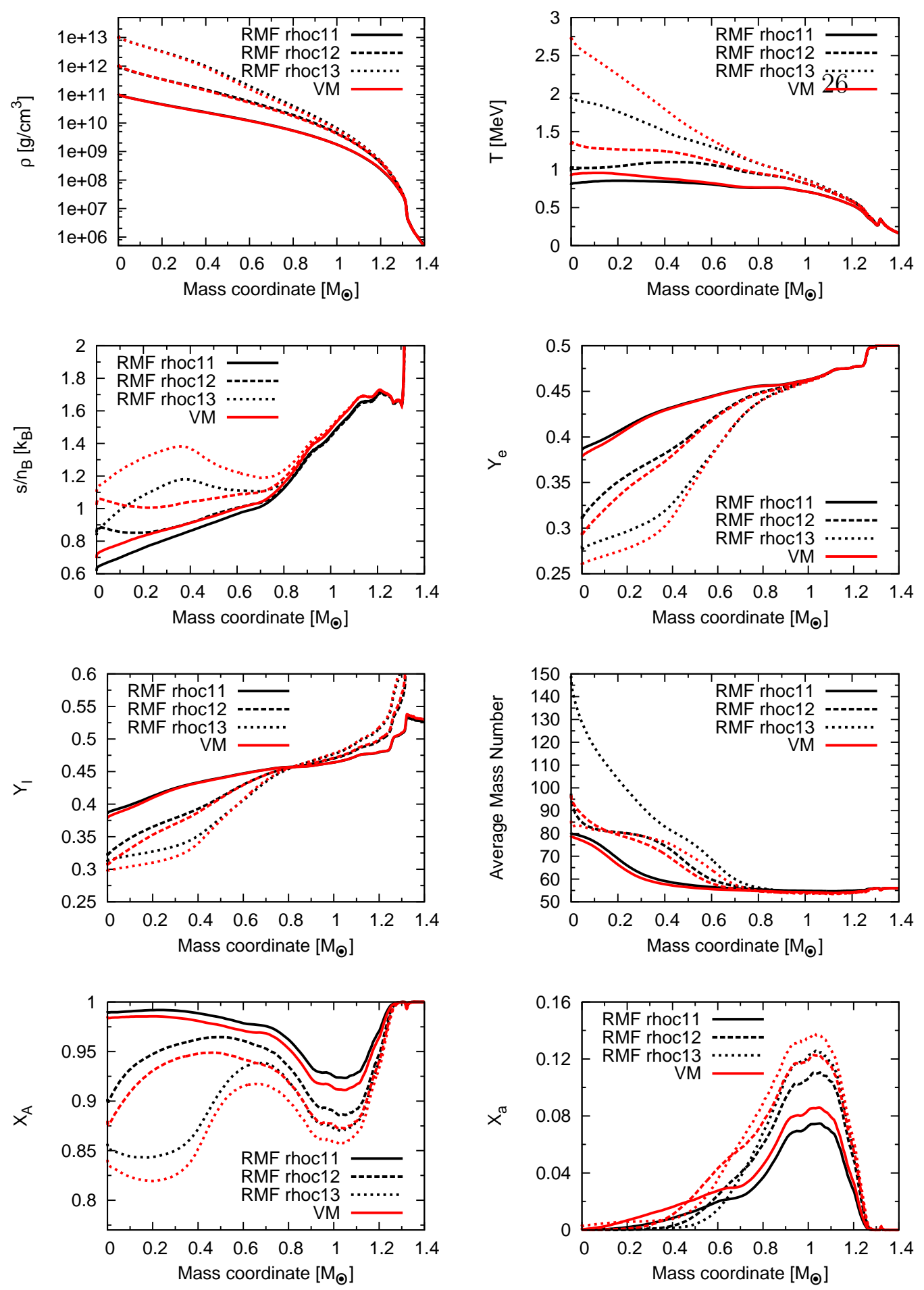

Figure 11. Radial distributions of several quantities of relevance obtained by 1D spherically symmetric simulations for the VM (red lines) and RMF (black lines) models at different times in the pre-bounce phase when the central density reaches $10^{11}$ (solid lines), $10^{12}$ (dashed lines) and $10^{13}$ (dotted lines) $\mathrm{g} / \mathrm{cm}^{3}$. The horizontal axis is the mass coordinate. 

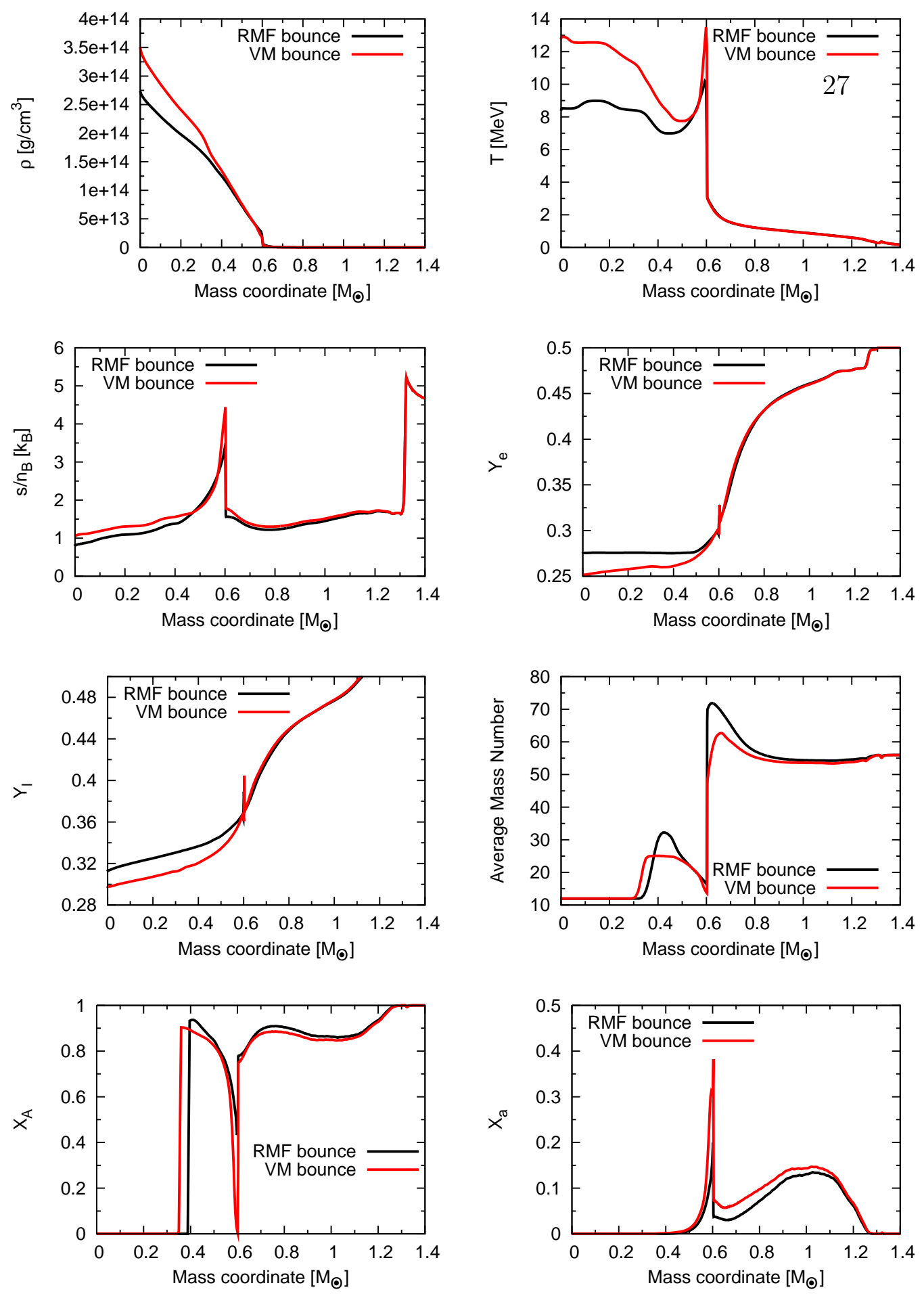

Figure 12. Same as Fig. 11 but at core bounce. 

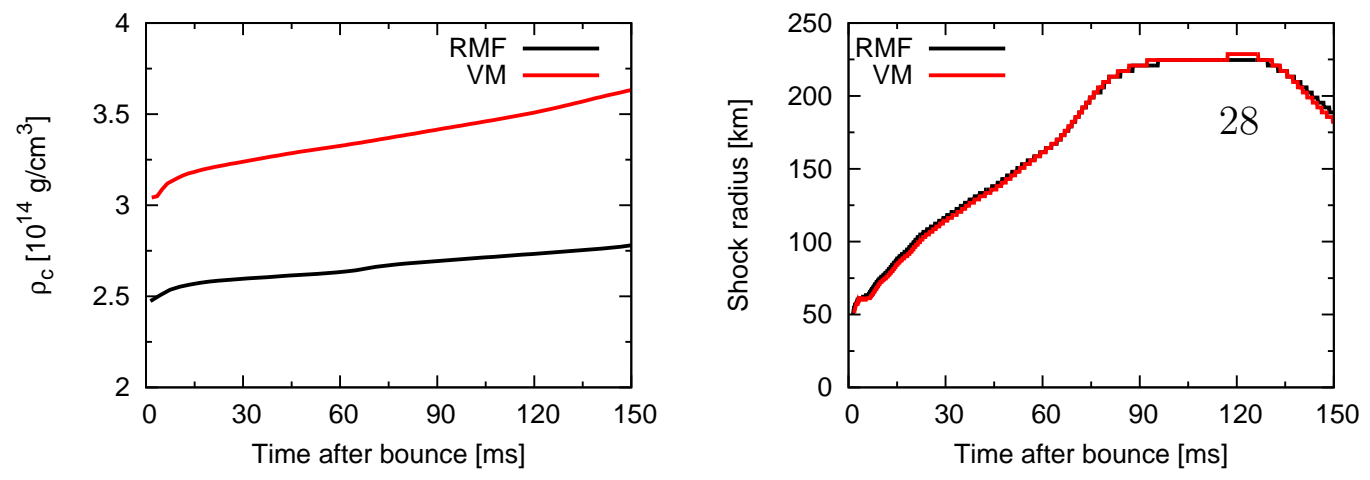

Figure 13. Time trajectories of the central density (left panel) and shock radius (right panel) for both models.
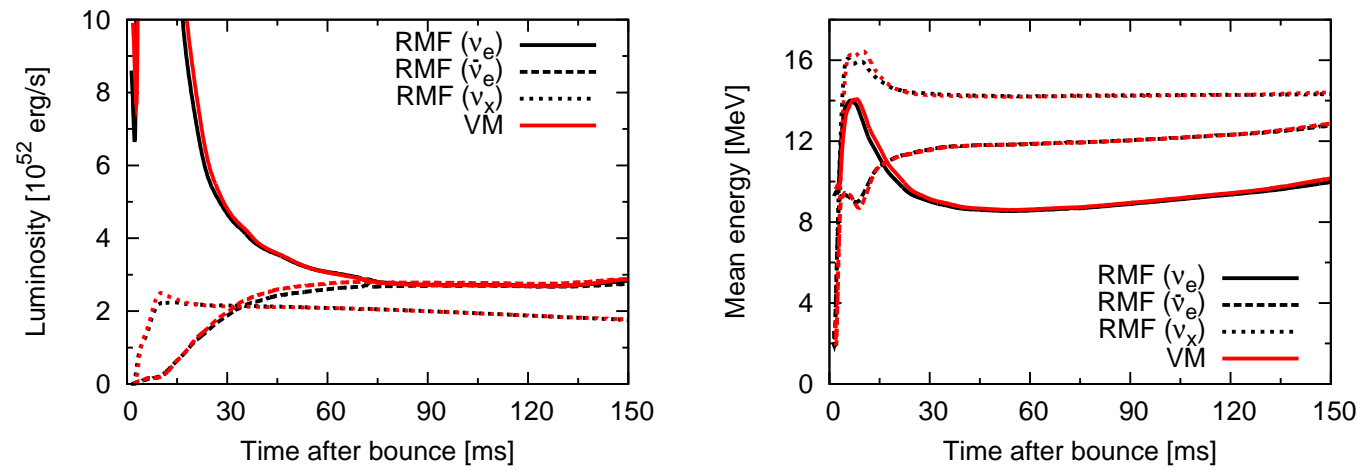

Figure 14. Time evolutions of the luminosities (left panel) and mean energies (right panel) of neutrinos. Note that the mean energy is defined as the ratio of the energy density to the number density of neutrinos.
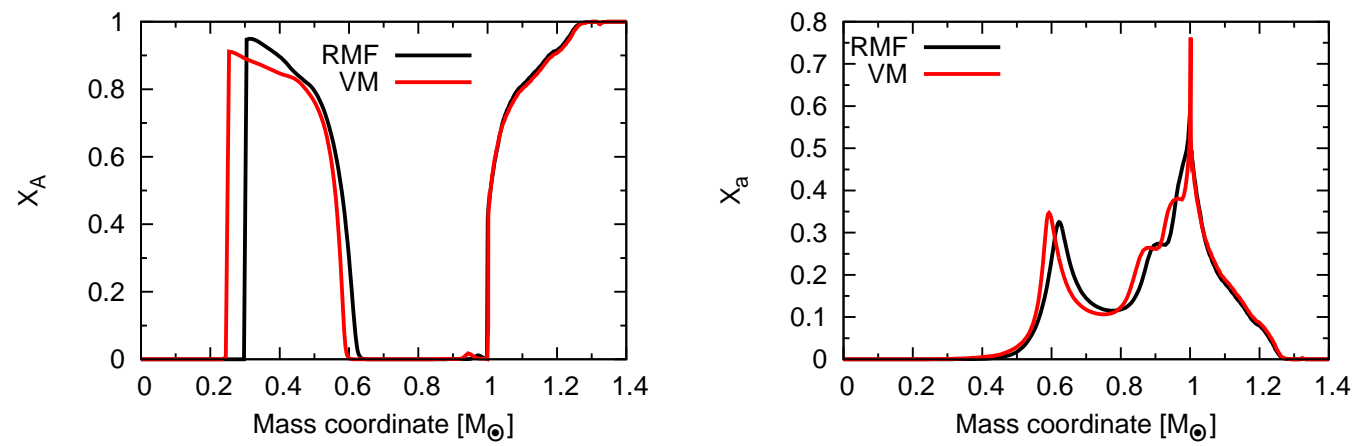

Figure 15. Radial distributions of the mass fraction of heavy (left) and light (right) nuclei at the time when the shock reaches $1.0 \mathrm{M}_{\odot}$ on the mass coordinate for the VM and RMF models. 

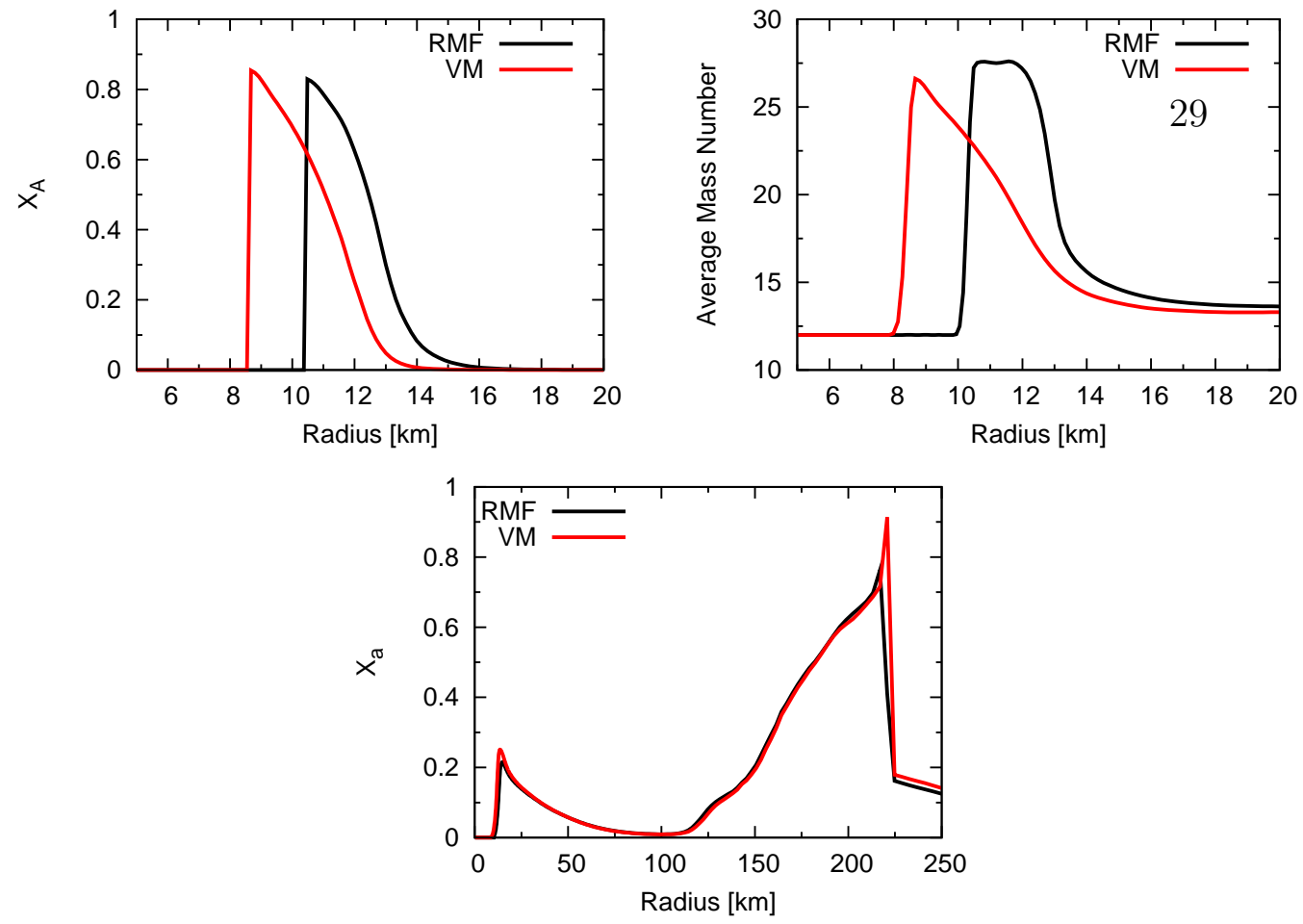

Figure 16. Radial distributions of the mass fraction of heavy (top left) and light (bottom) nuclei and the average mass numbers (top right) for the VM (red lines) and RMF (black lines) models at $100 \mathrm{~ms}$ after core bounce. Only the vicinity of the PNS surface $(5-20 \mathrm{~km})$ is shown in the top panels whereas the entire post-shock region is exhibited in the bottom panel. 